\title{
SYMMETRY AND EXISTENCE OF SOLUTIONS OF SEMI-LINEAR ELLIPTIC SYSTEMS IN HYPERBOLIC SPACE
}

\author{
HAIYANG HE \\ College of Mathematics and Computer Science, \\ Key Laboratory of High Performance Computing and Stochastic Information Processing \\ (Ministry of Education of China), Hunan Normal University, \\ Changsha, Hunan 410081, P.R. China \\ e-mail: hehy917@hotmail.com
}

(Received 4 September 2013; revised 19 December 2013; accepted 27 January 2014; first published online 18 December 2014)

Abstract.

$$
\left\{\begin{aligned}
-\Delta_{\mathbb{H}^{N}} u & =|v|^{p-1} v \\
-\Delta_{\mathbb{W}^{N}} v & =|u|^{q-1} u
\end{aligned}\right.
$$

in the whole Hyperbolic space $\mathbb{H}^{N}$. We establish decay estimates and symmetry properties of positive solutions. Unlike the corresponding problem in Euclidean space $\mathbb{R}^{N}$, we prove that there is a positive solution pair $(u, v) \in H^{1}\left(\mathbb{M}^{N}\right) \times H^{1}\left(\mathbb{M}^{N}\right)$ of problem (0.1), moreover a ground state solution is obtained. Furthermore, we also prove that the above problem has a radial positive solution.

2010 Mathematics Subject Classification. 58J05, 35J60.

1. Introduction and main result. In this paper, we will study decay, symmetry and existence of positive solutions of the following semi-linear elliptic systems

$$
\left\{\begin{aligned}
-\Delta_{\mathbb{\boxplus}^{N}} u & =|v|^{p-1} v, \\
-\Delta_{\mathbb{\boxplus}^{N}} v & =|u|^{q-1} u,
\end{aligned}\right.
$$

on Hyperbolic space $\mathbb{H}^{N}$, where $\Delta_{\mathbb{H}^{N}}$ denotes the Laplace-Beltrami operator on $\mathbb{M}^{N}, N \geq 3$, and $p$ and $q$ satisfy a suitable condition.

When posed in the Euclidean space $\mathbb{R}^{N}$, problem (1.1) has two features. First, it is the Emden-Fowler equation,

$$
-\Delta u=|u|^{p-1} u \text { in } \mathbb{R}^{N}
$$

Such a problem has been extensively studied, see for instance $[\mathbf{9}, \mathbf{1 0}, 13-15]$ and references therein. There is a host of later important contributions to the subject, among them we must mention the famous paper [15], where the Liouville-type theorem of problem (1.2) was obtained. They proved that the only non-negative solution of (1.2) is $u=0$ when $1 \leq p<\frac{N+2}{N-2}, N \geq 3$. A simple generalization of (1.2) to the case of systems is the following model,

$$
\left\{\begin{array}{l}
-\Delta u=|v|^{p-1} v, \text { in } \mathbb{R}^{N}, \\
-\Delta v=|u|^{q-1} u, \text { in } \mathbb{R}^{N} .
\end{array}\right.
$$


Using a blow-up technique, Qing [17] and Souto [26] had established a priori estimates for the solutions of problem (1.3). In [11], De Figueiredo and Felmer proved that if $p>0, q>0$ are such that $p, q \leq \frac{N+2}{N-2}$, but not both are equal to $\frac{N+2}{N-2}$, then the only non-negative solution of (1.3) is the trivial one $u=0, v=0$. Second, it is the following problem,

$$
-\Delta u+u=|u|^{p-1} u \text { in } \mathbb{R}^{N}
$$

There are many results about the existence of non-trivial or positive solutions of (1.4). A typical method is to use the radially symmetric Sobolev space or concentrationcompactness technique by Lions [19]. However, for system such as (1.4) in $\mathbb{R}^{N}$, there are fewer results. De Figueiredo and Yang [12] showed the existence of a strong radial pair solution, but the uniqueness result is not known. For more general nonlinear elliptic equations in the Euclidean space $\mathbb{R}^{N}$, we refer to $[\mathbf{7}, \mathbf{8}, \mathbf{2 3}, \mathbf{2 4}]$ and references therein.

It is also of interest to study problems (1.2) and (1.3) with respect to different ambient geometries in particular to see how curvature properties affect the existence and nature of solutions. A recent paper by Mancini and Sandeep [22] has studied the existence/non-existence and uniqueness of positive solution of the following elliptic equation,

$$
-\Delta_{\llbracket^{N}} u=|u|^{p-1} u+\lambda u
$$

in the subcritical case for every $\lambda<\left(\frac{N-1}{2}\right)^{2}$ and in critical exponent case for $\frac{N(N-1)}{4}<$ $\lambda \leq\left(\frac{N-1}{2}\right)^{2}$ with $N \geq 4$ on Hyperbolic space $\mathbb{H}^{N}$. Moreover, they proved that if $\lambda=0$ and $1<p<\frac{N+2}{N-2}$, then problem (1.5) has a positive solution. This result is contrasted with the result in Euclidean space due to [15]. Afterwards, Bhakta and Sandeep [5] have investigated a priori estimates, existence/non-existence of radial sign changing solutions of problem (1.5). In [6], the classification of radial solutions of problem (1.5) is done by Bonforte et al. [6].

Thanks to the Poincaré inequality

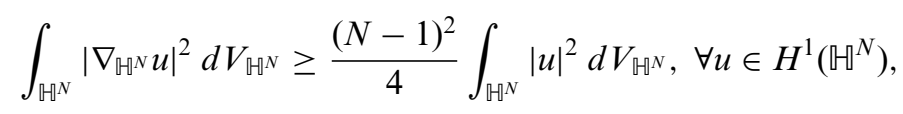

we can study the decay, symmetry properties of the positive solutions of problem (1.1). We also prove that problem (1.1) possesses at least a positive solution, which should be contrasted with the result due to Figueiredo and Felmer [11]. The difficulties in treating system (1.1) originate in at least three facts. First, there is a lack of compactness due to the fact that we are working in $\mathbb{T}^{N}$, which is a non-compact manifold. Second, due to the type of growth of the nonlinear term, we cannot work with the usual space $H^{1}\left(\mathbb{M}^{N}\right)$, and then we need inhomogeneous Sobolev space. Third, although problem (1.1) has a variational structure, the functional associated to it is strongly indefinite.

Now we are ready to state our main result. In Section 2, we discuss the symmetry property of positive solutions of problem (1.1).

THEOREM 1.1. For p, q satisfying

$$
1<p, q \leq \frac{N+2}{N-2}
$$


then all positive solutions $(u, v) \in H^{1}\left(\mathbb{H}^{N}\right) \times H^{1}\left(\mathbb{H}^{N}\right)$ of problem (1.1) are hyperbolic symmetry, i.e. there is $x_{0} \in \mathbb{H}^{N}$ such that $(u, v)$ is constant on hyperbolic spheres centered at $x_{0}$.

In Section 3, we prove a result on the decay of positive solutions of problem (1.1) as $|x| \rightarrow \infty$ and, in fact, exponential decay. The results of Section 3 are the following.

THEOREM 1.2. Suppose that p, q satisfy (1.6), and $(u, v) \in H^{1}\left(\mathbb{H}^{N}\right) \times H^{1}\left(\mathbb{M}^{N}\right)$ be a positive radial solution of (1.1). Then $u^{\prime}(r)<0, v^{\prime}(r)<0$ for $r>0$ and

$$
\lim _{r \rightarrow \infty} u(r)=\lim _{r \rightarrow \infty} v(r)=\lim _{r \rightarrow \infty} u^{\prime}(r)=\lim _{r \rightarrow \infty} v^{\prime}(r)=0 .
$$

Moreover,

$$
\lim _{r \rightarrow \infty} \frac{\log u^{2}}{r}=\lim _{r \rightarrow \infty} \frac{\log v^{2}}{r}=\lim _{r \rightarrow \infty} \frac{\log u^{\prime 2}}{r}=\lim _{r \rightarrow \infty} \frac{\log v^{\prime 2}}{r}=-2(N-1) .
$$

In Section 4, we prove the existence of radial symmetric positive solutions of problem (1.1). We also obtain the existence of a radial ground state positive solution for system (1.1). That is,

THEOREM 1.3. Suppose that $(p, q)$ satisfies

$$
\frac{1}{p+1}+\frac{1}{q+1}>\frac{N-2}{N},
$$

then problem (1.1) has at least one radial positive solution $(u, v)$.

THEOREM 1.4. Suppose that $1<p, q<\frac{N+2}{N-2}$, then problem (1.1) has a radial positive ground state solution.

Finally, in Section 5, using the concentration function, we study the existence of solutions of problem (1.1), which belongs to $H^{1}\left(\mathbb{H}^{N}\right) \times H^{1}\left(\mathbb{H}^{N}\right)$. Moreover, a ground state solution for system (1.1) is also obtained.

THEOREM 1.5. Suppose that $1<p, q<\frac{N+2}{N-2}$, then problem (1.1) has a positive solution that belongs to $H^{1}\left(\mathbb{H}^{N}\right) \times H^{1}\left(\mathbb{M}^{N}\right)$. Moreover, there is a ground state solution for system (1.1).

2. Proof of Theorem 1.1. The main purpose of this section is to prove hyperbolic symmetry properties of positive solutions of (1.1). The way of proving this symmetry is by the move plane method, as originally introduced by Alexandroff $[\mathbf{1 , 1 6}]$, later used by Serrin [25], and extensively used in recent times after the work of Gidas-Ni-Nirenberg [13]. The case on non-compact manifold was studied in $[2,3]$.

Proof of Theorem 1.1. This proof is in the spirit of [2]. Let $A_{t}$ be a one-parameter group of isometries of $\mathbb{W}^{N}$, which is $C^{1}\left(\mathbb{R} \times \mathbb{H}^{N}, \mathbb{W}^{N}\right)$, and $I$ be a reflection (i.e. $I$ is an isometry and $I^{2}=$ Identity) satisfying the invariance condition

$$
A_{t} I A_{t}=I, \forall t \in \mathbb{R} .
$$


We translate the reflection $I$ using $A_{t}$ to define a one-parameter family of reflections,

$$
I_{t}=A_{t} I A_{-t} .
$$

Let $U_{t}$ be the hyper-surface of $\mathbb{H}^{N}$, which is fixed by $I_{t}$. We also assume that $\bigcup_{t_{1}<t<t_{2}} U_{t}$ is open for all $t_{1}, t_{2} \in \mathbb{R}$ and $\bigcup_{t \in \mathbb{R}} U_{t}=\mathbb{U}^{N}$. For $t \in \mathbb{R}$, define

$$
Q_{t}=\bigcup_{-\infty<s<t} U_{s}, \quad Q^{t}=\bigcup_{t<s<\infty} U_{s}
$$

then we have $I_{t}\left(Q_{t}\right) \subset Q^{t}$, and $I_{t}\left(Q^{t}\right) \subset Q_{t}$ for all $t \in \mathbb{R}$. For $t \in \mathbb{R}$ and $x \in Q_{t}$, we define $x_{t}=I_{t}(x)$, and

$$
u_{t}(x)=u\left(I_{t}(x)\right), v_{t}(x)=v\left(I_{t}(x)\right)
$$

Define

$$
\Lambda=\left\{t \in \mathbb{R}: \forall \tau>t, u \geq u_{\tau}, \text { and } v \geq v_{\tau}, \text { in } Q_{\tau}\right\},
$$

the first step of proof is to show that set $\Lambda$ is non-empty, the second step is to prove that $\Lambda$ is bounded from below. Finally we will show that if $\bar{\Lambda}=\inf \Lambda$, then $u \equiv u_{\bar{\Lambda}}, v \equiv v_{\bar{\Lambda}}$ in $Q_{\bar{\Lambda}}$.

Step 1. $\Lambda$ is non-empty.

Since $(u, v) \in H^{1}\left(\mathbb{H}^{N}\right) \times H^{1}\left(\mathbb{H}^{N}\right)$, we can take $\left(u_{\lambda}-u\right)^{+}$or $\left(v_{\lambda}-v\right)^{+}$as test function. Then we obtain

$$
\begin{aligned}
\int_{Q_{\lambda}}\left|\nabla_{\mathbb{H}^{N}}\left(u_{\lambda}-u\right)^{+}\right|^{2} d V_{\mathbb{H}^{N}} & =\int_{Q_{\lambda}}\left(\left|v_{\lambda}\right|^{p-1} v_{\lambda}-|v|^{p-1} v\right)\left(u_{\lambda}-u\right)^{+} d V_{\mathbb{H}^{N}} \\
\leq & \left(\int_{Q_{\lambda}}\left|v_{\lambda}\right|^{p+1} d V_{\mathbb{H}^{N}}\right)^{\frac{p-1}{p+1}}\left(\int_{Q_{\lambda}}\left|\left(v_{\lambda}-v\right)^{+}\right|^{p+1} d V_{\mathbb{H}^{N}}\right)^{\frac{1}{p+1}}\left(\int_{Q_{\lambda}}\left|\left(u_{\lambda}-u\right)^{+}\right|^{p+1} d V_{\mathbb{H}^{N}}\right)^{\frac{1}{p+1}} \\
\quad \leq & c\left(\int_{Q_{\lambda}}\left|v_{\lambda}\right|^{p+1} d V_{\mathbb{H}^{N}}\right)^{\frac{p-1}{p+1}}\left(\int_{Q_{\lambda}}\left|\nabla_{\mathbb{H}^{N}}\left(v_{\lambda}-v\right)^{+}\right|^{2} d V_{\mathbb{H}^{N}}\right)^{\frac{1}{2}}\left(\int_{Q_{\lambda}}\left|\nabla_{\mathbb{H}^{N}}\left(u_{\lambda}-u\right)^{+}\right|^{2} d V_{\mathbb{H}^{N}}\right)^{\frac{1}{2}},
\end{aligned}
$$

and

$$
\begin{aligned}
& \int_{Q_{\lambda}}\left|\nabla_{\mathbb{H}^{N}}\left(v_{\lambda}-v\right)^{+}\right|^{2} d V_{\mathbb{\boxplus}^{N}} \\
& \quad \leq c\left(\int_{Q_{\lambda}}\left|u_{\lambda}\right|^{q+1} d V_{\mathbb{H}^{N}}\right)^{\frac{q-1}{q+1}}\left(\int_{Q_{\lambda}}\left|\nabla_{\mathbb{H}^{N}}\left(u_{\lambda}-u\right)^{+}\right|^{2} d V_{\mathbb{H}^{N}}\right)^{\frac{1}{2}}\left(\int_{Q_{\lambda}}\left|\nabla_{\mathbb{H}^{N}}\left(v_{\lambda}-v\right)^{+}\right|^{2} d V_{\mathbb{H}^{N}}\right)^{\frac{1}{2}} .
\end{aligned}
$$

It implies that

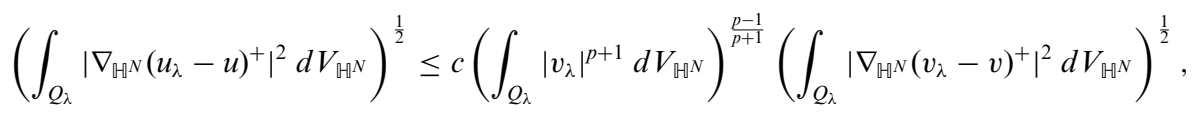

and

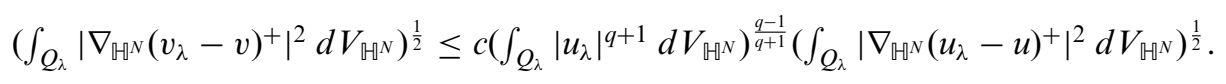


Thus,

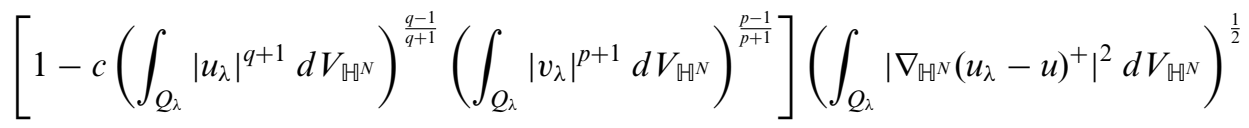

$$
\begin{aligned}
& \leq 0 \text {, }
\end{aligned}
$$

and

$$
\begin{aligned}
& {\left[1-c\left(\int_{Q_{\lambda}}\left|u_{\lambda}\right|^{q+1} d V_{\mathbb{\boxplus}^{N}}\right)^{\frac{q-1}{q+1}}\left(\int_{Q_{\lambda}}\left|v_{\lambda}\right|^{p+1} d V_{\mathbb{U}^{N}}\right)^{\frac{p-1}{p+1}}\right]\left(\int_{Q_{\lambda}}\left|\nabla_{\mathbb{\boxplus}^{N}}\left(v_{\lambda}-v\right)^{+}\right|^{2} d V_{\mathbb{U}^{N}}\right)^{\frac{1}{2}}} \\
& \leq 0 \text {. }
\end{aligned}
$$

Since we can choose $\lambda_{1} \in \mathbb{R}$, such that

$$
c\left(\int_{Q_{\lambda}}\left|u_{\lambda}\right|^{q+1} d V_{\mathbb{\llbracket} N}\right)^{\frac{q-1}{q+1}}\left(\int_{Q_{\lambda}}\left|v_{\lambda}\right|^{p+1} d V_{\mathbb{\square} N}\right)^{\frac{p-1}{p+1}}<1,
$$

for any $\lambda>\lambda_{1}$, we have

$$
\int_{Q_{\lambda}}\left|\nabla_{\mathfrak{M}^{N}}\left(u_{\lambda}-u\right)^{+}\right|^{2} d V_{\mathbb{M}^{N}} \leq 0,
$$

and

$$
\int_{Q_{\lambda}}\left|\nabla_{\mathbb{H}^{N}}\left(v_{\lambda}-v\right)^{+}\right|^{2} d V_{\mathbb{\boxplus}^{N}} \leq 0 .
$$

Therefore, $\left(u_{\lambda}-u\right)^{+} \equiv 0$, and $\left(v_{\lambda}-v\right)^{+} \equiv 0$ in $Q_{\lambda}$, and $\left(\lambda_{1},+\infty\right) \subset \Lambda$.

Step 2. $\Lambda$ is bounded from below.

By $(u, v) \in H^{1}\left(\mathbb{M}^{N}\right) \times H^{1}\left(\mathbb{W}^{N}\right)$, we have

$$
\lim _{\lambda \rightarrow-\infty} \sup _{Q_{\lambda}} u=\lim _{\lambda \rightarrow-\infty} \sup _{Q_{\lambda}} v=0,
$$

and we may choose $\lambda_{2}$ such that

$$
\sup _{Q_{\lambda_{2}} u<\frac{\sup ^{N} u}{2},} \sup _{Q_{\lambda_{2}}} v<\frac{\sup _{\mathbb{\boxplus}^{N}} v}{2} .
$$

This implies that all $\lambda \in\left(-\infty, \lambda_{2}\right)$ do not belong to $\Lambda$. Therefore, $\Lambda$ is bounded from below, and we let $\bar{\Lambda}=\inf \Lambda$.

Step 3. $u \equiv u_{\bar{\Lambda}}, v \equiv v_{\bar{\Lambda}}$, in $Q_{\bar{\Lambda}}$.

In fact, it is clear that by the continuity of the foliation of $(u, v)$, we have

$$
u \geq u_{\bar{\Lambda}}, v \geq v_{\bar{\Lambda}}, \text { in } Q_{\bar{\Lambda}} .
$$


Now observing that if $u \equiv u_{\bar{\Lambda}}$, it follows from

$$
\left\{\begin{array}{l}
-\Delta_{\llbracket^{N}}\left(u-u_{\bar{\Lambda}}\right)=|v|^{p-1} v-\left|v_{\bar{\Lambda}}\right|^{p-1} v_{\bar{\Lambda}}, \\
-\Delta_{\mathbb{\boxplus}^{N}}\left(v-v_{\bar{\Lambda}}\right)=|u|^{q-1} u-\left|u_{\bar{\Lambda}}\right|^{q-1} u_{\bar{\Lambda}},
\end{array}\right.
$$

we can get $v \equiv v_{\bar{\Lambda}}$. So if we assume by contradiction that step 3 is not true, we conclude that

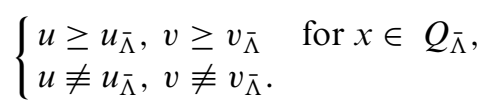

The strong maximum principle and connectedness of $Q_{\bar{\Lambda}}$ imply that

$$
\left\{\begin{array}{l}
u>u_{\bar{\Lambda}}, \text { for } x \in Q_{\bar{\Lambda}}, \\
v>v_{\bar{\Lambda}}, \text { for } x \in Q_{\bar{\Lambda}},
\end{array}\right.
$$

and

$$
\left\{\begin{array}{l}
X(u)(x)>0, \text { for } x \in U_{\bar{\Lambda}}, \\
X(v)(x)>0, \text { for } x \in U_{\bar{\Lambda}},
\end{array}\right.
$$

where $X$ is the vector field associated to the transformation group $A_{t}$, we shall see that this is impossible.

Choose $x_{1} \in U_{\bar{\Lambda}}$, and $R_{0}>0$. By the continuity of foliation, there would exist $\varepsilon_{0}>$ 0 such that for $0<\varepsilon<\varepsilon_{0}, I_{\bar{\Lambda}-\varepsilon}\left(B\left(x_{1}, R_{0}\right)\right) \subset B\left(x_{1}, 2 R_{0}\right)$. Moreover, by the definition of $\bar{\Lambda}$, we could construct an increasing sequence $\lambda_{n} \leq \bar{\Lambda}$ such that $\lambda_{n}>\bar{\Lambda}-\varepsilon_{0}$, and $\exists y_{n} \in Q_{\lambda_{n}}$ such that

$$
u\left(y_{n}\right)<u\left(I_{\lambda_{n}}\left(y_{n}\right)\right)=u_{\lambda_{n}}\left(y_{n}\right)
$$

or

$$
v\left(y_{n}\right)<v\left(I_{\lambda_{n}}\left(y_{n}\right)\right)=v_{\lambda_{n}}\left(y_{n}\right) .
$$

We claim that $y_{n} \in B\left(x_{1}, 2 R_{0}\right)$. If it is not true, we can take $\left(u_{\lambda_{n}}-u\right)^{+}$or $\left(v_{\lambda_{n}}-v\right)^{+}$ as a test function (as in the first step). We would have that $u_{\lambda_{n}} \leq u$, or $v_{\lambda_{n}} \leq v$, in $Q_{\lambda_{n}}$. This proves our claim.

Modulo a subsequence. There would exist $y \in Q_{\bar{\Lambda}}$, such that $y_{n} \longrightarrow y$. By continuity we have

$$
u(y)=\lim _{n \rightarrow+\infty} u\left(y_{n}\right) \leq \lim _{n \rightarrow+\infty} u_{\lambda_{n}}\left(y_{n}\right)=u_{\bar{\Lambda}}(y),
$$

or

$$
v(y)=\lim _{n \rightarrow+\infty} v\left(y_{n}\right) \leq \lim _{n \rightarrow+\infty} v_{\lambda_{n}}\left(y_{n}\right)=v_{\bar{\Lambda}}(y) .
$$

It implies that $y \in U_{\bar{\Lambda}}$. On the other hand, there exist points $\xi_{n}$ in the line segment between $y_{n}$ and $I_{\lambda_{n}}\left(y_{n}\right)$ such that $X(u)\left(\xi_{n}\right) \leq 0$, passing to the limit we should have $X(u)(y) \leq 0$. This is impossible, since $X(u)(x)>0$ for all $x \in U_{\bar{\Lambda}}$. Hence, $u \equiv u_{\bar{\Lambda}}$, and $v \equiv v_{\bar{\Lambda}}$, in $Q_{\bar{\Lambda}}$. 
3. Decay estimates. The hyperbolic $\mathrm{N}$-space $\mathbb{H}^{N}, N \geq 2$ is a complete simple connected Riemannian manifold having constant sectional curvature equal to -1 , and for a given dimensional number, any two such spaces are isometric [28]. There are several models for $\mathbb{M}^{N}$, the most important being the half-space model, the ball model and the hyperboloid or Lorentz model.

Let $\mathbb{B}^{N}=\left\{x \in \mathbb{R}^{N}:|x|<1\right\}$ denote the unit disc in $\mathbb{R}^{N}$. The space $\mathbb{H}^{N}$ endowed with the Riemannian metric $g$ given by $g_{i j}=(p(x))^{2} \delta_{i j}$ is called the ball model of the hyperbolic space, where $p(x)=\frac{2}{1-|x|^{2}}$. The hyperbolic gradient and the LaplaceBeltrami operator are:

$$
\begin{aligned}
\Delta_{\mathbb{\boxplus}^{N}} & \left.=\left(\frac{1-|x|^{2}}{2}\right)^{2} \Delta+(N-2) \frac{1-|x|^{2}}{2}\langle x, \nabla\rangle=(p(x))^{-N} \operatorname{div}\left((p(x))^{N-2} \nabla\right)\right), \\
\nabla_{\mathbb{\boxplus}^{N}} u & =\frac{\nabla u}{p(x)},
\end{aligned}
$$

where $\nabla$ and div denote the Euclidean gradient and divergence in $\mathbb{R}^{N}$ respectively. Let $H^{1}\left(\mathbb{H}^{N}\right)$ denote the Sobolev space on $\mathbb{H}^{N}$ with the above metric $g$, then we have $H^{1}\left(\mathbb{M}^{N}\right) \hookrightarrow L^{p}\left(\mathbb{M}^{N}\right)$ for $2 \leq p \leq \frac{2 N}{N-2}$ when $N \geq 3$ and $p \geq 2$ when $N=2$. From the Poincaré inequality

$$
\int_{\mathbb{\mathbb { H } ^ { N }}}\left|\nabla_{\mathbb{U}^{N}} u\right|^{2} d V_{\mathbb{\boxplus}^{N}} \geq \frac{(N-1)^{2}}{4} \int_{\mathbb{G}^{N}}|u|^{2} d V_{\mathbb{\boxplus}^{N}}, \quad \forall u \in H^{1}\left(\mathbb{\boxplus}^{N}\right),
$$

we know that $\int_{\mathbb{H} \mathbb{H}^{N}}\left|\nabla_{\mathbb{H}^{N}} u\right|^{2} d V_{\mathbb{H}^{N}}$ is a norm equivalent to $H^{1}\left(\mathbb{H}^{N}\right)$.

In this section, the proof of these decay estimates closely follow the proof of the decay estimates proved in [22]. Let $(u, v)$ be a positive symmetric solution of problem (1.1), and $u=u(|\xi|), v=v(|\xi|),|\xi|<1$, then

$$
\left\{\begin{array}{l}
\left(\frac{1-|\xi|^{2}}{2}\right)^{2} \Delta u+(N-2) \frac{1-|\xi|^{2}}{2}\langle\xi, \nabla u\rangle+v^{p}=0 \\
\left(\frac{1-|\xi|^{2}}{2}\right)^{2} \Delta v+(N-2) \frac{1-|\xi|^{2}}{2}\langle\xi, \nabla v\rangle+u^{q}=0
\end{array}\right.
$$

Setting $|\xi|=\tanh \frac{t}{2}, u(t)=u\left(\tanh \frac{t}{2}\right), v(t)=v\left(\tanh \frac{t}{2}\right), k(t)=(\sinh t)^{N-1}$, it is easy to see that

$$
\begin{aligned}
& \int_{\mathbb{\boxplus}^{N}}|u|^{q+1} d V_{\mathbb{\boxplus}^{N}}=w_{N-1} \int_{0}^{\infty} k(t)|u|^{q+1} d t, \\
& \int_{\mathbb{H}^{N}}|v|^{p+1} d V_{\mathbb{H}^{N}}=w_{N-1} \int_{0}^{\infty} k(t)|v|^{p+1} d t, \\
& \int_{\mathbb{\mathbb { H } ^ { N }}}\left|\nabla_{\mathbb{H}^{N}} u\right|^{2} d V_{\mathbb{\boxplus}^{N}}=w_{N-1} \int_{0}^{\infty} k(t)\left|u^{\prime}\right|^{2} d t, \\
& \int_{\mathbb{\boxplus}^{N}}\left|\nabla_{\mathbb{U}^{N}} v\right|^{2} d V_{\mathbb{\boxplus}^{N}}=w_{N-1} \int_{0}^{\infty} k(t)\left|v^{\prime}\right|^{2} d t,
\end{aligned}
$$

where $w_{N-1}$ denotes the surface area of $S^{N-1}$. 
In addition, (3.1) is rewritten as

$$
\left\{\begin{array}{l}
u^{\prime \prime}+\frac{N-1}{\tanh t} u^{\prime}+v^{p}=0, \\
v^{\prime \prime}+\frac{N-1}{\tanh t} v^{\prime}+u^{q}=0 \\
u^{\prime}(0)=v^{\prime}(0)=0,
\end{array}\right.
$$

as well as

$$
\left\{\begin{array}{l}
\left(k(t) u^{\prime}\right)^{\prime}+k(t) v^{p}=0, \\
\left(k(t) v^{\prime}\right)^{\prime}+k(t) u^{q}=0, \\
u^{\prime}(0)=v^{\prime}(0)=0,
\end{array}\right.
$$

and if $(u, v) \in H^{1}\left(\mathbb{M}^{N}\right) \times H^{1}\left(\mathbb{M}^{N}\right)$ solves (3.1), then

$$
\int_{0}^{\infty} k(t) u^{\prime} v^{\prime} d t=\int_{0}^{\infty} k(t) u^{q+1} d t=\int_{0}^{\infty} k(t) v^{p+1} d t .
$$

Now, note that $(u, v)$ solves (3.2), and

$$
J_{(u, v)}(t)=u^{\prime} v^{\prime}+\frac{|v|^{p+1}}{p+1}+\frac{|u|^{q+1}}{q+1},
$$

then

$$
\begin{aligned}
\frac{d}{d t} J_{(u, v)}(t) & =u^{\prime \prime} v^{\prime}+u^{\prime} v^{\prime \prime}+v^{\prime} v^{p}+u^{\prime} u^{q}=\left(u^{\prime \prime}+v^{p}\right) v^{\prime}+u^{\prime}\left(v^{\prime \prime}+u^{q}\right) \\
& =-\frac{N-1}{\tanh t} v^{\prime 2}-\frac{N-1}{\tanh t} u^{\prime 2} \leq 0 . \quad \forall t>0 .
\end{aligned}
$$

LeMma 3.1. Let $(u, v) \in H^{1}\left(\mathbb{H}^{N}\right) \times H^{1}\left(\mathbb{H}^{N}\right)$ be a positive solution of (3.2), then $u^{\prime}(t)<0, v^{\prime}(t)<0$, for every $t>0$, and

$$
\lim _{t \rightarrow+\infty} u(t)=\lim _{t \rightarrow+\infty} v(t)=\lim _{t \rightarrow+\infty} u^{\prime}(t)=\lim _{t \rightarrow+\infty} v^{\prime}(t)=0 .
$$

Proof. By equation (3.3), we have $\left(k(t) u^{\prime}(t)\right)^{\prime}<0$, and $\left(k(t) v^{\prime}(t)\right)^{\prime}<0$, and from $u^{\prime}(0)=v^{\prime}(0)=0$, then $u^{\prime}(t)<0, v^{\prime}(t)<0, \forall t>0$.

In particular, by $u(t)>0, v(t)>0$, it exists

$$
u(\infty)=\lim _{t \rightarrow+\infty} u(t), \quad v(\infty)=\lim _{t \rightarrow+\infty} v(t) .
$$

Since $J_{(u . v)}$ is decreasing, $\lim _{t \rightarrow+\infty} u^{\prime}(t) v^{\prime}(t)$ exists.

Since $(u, v) \in H^{1}\left(\mathbb{H}^{N}\right) \times H^{1}\left(\mathbb{H}^{N}\right)$, we have

$$
\liminf _{t \rightarrow+\infty} k(t)\left[u^{\prime 2}(t)+u^{2}(t)\right]=0,
$$

and

$$
\liminf _{t \rightarrow+\infty} k(t)\left[v^{\prime 2}(t)+v^{2}(t)\right]=0 .
$$


Thus, we have that

$$
\lim _{t \rightarrow+\infty} u(t)=\lim _{t \rightarrow+\infty} v(t)=\lim _{t \rightarrow+\infty} u^{\prime}(t)=\lim _{t \rightarrow+\infty} v^{\prime}(t)=0
$$

LEMMA 3.2. Let $(u, v)$ be a positive solution of problem (1.1), and $(u, v) \in H^{1}\left(\mathbb{H}^{N}\right) \times$ $H^{1}\left(\mathbb{H}^{N}\right)$. Then

$$
\lim _{t \rightarrow+\infty} \frac{\log u^{2}}{t}=\lim _{t \rightarrow+\infty} \frac{\log v^{2}}{t}=\lim _{t \rightarrow+\infty} \frac{\log u^{\prime 2}}{t}=\lim _{t \rightarrow+\infty} \frac{\log v^{\prime 2}}{t}=-2(N-1) .
$$

Proof. By Lemma 3.1, we obtain

$$
\lim _{t \rightarrow+\infty} u(t)=\lim _{t \rightarrow+\infty} v(t)=0 .
$$

Then it exists that $t_{\varepsilon}>0$, such that

$$
\operatorname{coth} t \leq 1+\varepsilon, v^{p}(t) \leq \varepsilon v(t), u^{q}(t) \leq \varepsilon u(t), \quad \forall t \geq t_{\varepsilon} .
$$

Since $u^{\prime}(t)<0, v^{\prime}(t)<0$, we have for $t \geq t_{\varepsilon}$,

$$
\left\{\begin{array}{l}
u^{\prime \prime}+(N-1)(1+\varepsilon) u^{\prime} \leq u^{\prime \prime}+(N-1) \operatorname{coth} t u^{\prime}+v^{p}=0 \\
u^{\prime \prime}+(N-1) u^{\prime}+\varepsilon v \geq u^{\prime \prime}+(N-1) \operatorname{coth} t u^{\prime}+v^{p}=0
\end{array}\right.
$$

and

$$
\left\{\begin{array}{l}
v^{\prime \prime}+(N-1)(1+\varepsilon) v^{\prime} \leq v^{\prime \prime}+(N-1) \operatorname{coth} t v^{\prime}+u^{q}=0 \\
v^{\prime \prime}+(N-1) v^{\prime}+\varepsilon u \geq v^{\prime \prime}+(N-1) \operatorname{coth} t v^{\prime}+u^{q}=0
\end{array}\right.
$$

Then we get

$$
\begin{gathered}
(u+v)^{\prime \prime}+(N-1)(1+\varepsilon)(u+v)^{\prime} \leq(u+v)^{\prime \prime}+(N-1) \operatorname{coth} t(u+v)^{\prime}+u^{q}+v^{p}=0, \\
(u+v)^{\prime \prime}+(N-1)(u+v)^{\prime}+\varepsilon(u+v) \geq(u+v)^{\prime \prime}+(N-1) \operatorname{coth} t(u+v)^{\prime}+u^{q}+v^{p}=0 .
\end{gathered}
$$

It implies that

$$
(u+v)^{\prime \prime}+(N-1)(1+\varepsilon)(u+v)^{\prime} \leq(u+v)^{\prime \prime}+(N-1)(u+v)^{\prime}+\varepsilon(u+v) .
$$

Let $\mu^{-}(\varepsilon)=-(N-1)(1+\varepsilon), \mu^{+}(\varepsilon)=0, \quad$ and $\quad \nu^{-}(\varepsilon)=\frac{-(N-1)-\sqrt{(N-1)^{2}-4 \varepsilon}}{2}$, $v^{+}(\varepsilon)=\frac{-(N-1)+\sqrt{(N-1)^{2}-4 \varepsilon}}{2}$ be the characteristic roots of the differential Polinamials on the LHS and RHS of (3.6) respectively. We choose $\varepsilon<\frac{(N-1)^{2}}{4}$, then $v^{ \pm}(\varepsilon)$ is real and distinct. As in [22], we get

$$
\begin{gathered}
\left\{\begin{array}{l}
u(t)+v(t) \geq\left(\left[u\left(t_{\varepsilon}\right)+v\left(t_{\varepsilon}\right) e^{-\mu^{-}(\varepsilon) t_{\varepsilon}}\right]\right) e^{\mu^{-}(\varepsilon) t}, \quad \forall t \geq t_{\varepsilon}, \\
u(t)+v(t) \leq\left(\left[u\left(t_{\varepsilon}\right)+v\left(t_{\varepsilon}\right) e^{-v^{-}(\varepsilon) t_{\varepsilon}}\right]\right) e^{v^{-}(\varepsilon) t}, \quad \forall t \geq t_{\varepsilon},
\end{array}\right. \\
\left\{\begin{array}{l}
u^{\prime}(\tau)+v^{\prime}(\tau) \geq \mu^{-}(\varepsilon)(u(\tau)+v(\tau)), \quad \forall \tau \geq t_{\varepsilon}, \\
u^{\prime}(\tau)+v^{\prime}(\tau) \leq v^{-}(\varepsilon)(u(\tau)+v(\tau)), \quad \forall \tau \geq t_{\varepsilon} .
\end{array}\right.
\end{gathered}
$$


We see from (3.7) that

$$
2 \mu^{-}(\varepsilon) \leq \liminf _{t \rightarrow+\infty} \frac{\log (u+v)^{2}}{t} \leq \limsup _{t \rightarrow+\infty} \frac{\log (u+v)^{2}}{t} \leq 2 v^{-}(\varepsilon), \quad \forall \varepsilon>0,
$$

and hence,

$$
\lim _{t \rightarrow+\infty} \frac{\log (u+v)^{2}}{t}=-2(N-1) .
$$

From (3.7) and (3.8), we also get

$$
\lim _{t \rightarrow+\infty} \frac{\log \left(u^{\prime}+v^{\prime}\right)^{2}}{t}=-2(N-1)
$$

Since $u^{\prime}(t)<0, v^{\prime}(t)<0$, we conclude from (3.10) that

$$
\lim _{t \rightarrow+\infty} \frac{\log u^{\prime 2}}{t}=-2(N-1)
$$

and

$$
\lim _{t \rightarrow+\infty} \frac{\log v^{\prime 2}}{t}=-2(N-1) .
$$

From $u(t)>0, v(t)>0$, and (3.9), we also have that

$$
\lim _{t \rightarrow+\infty} \frac{\log u^{2}}{t}=\lim _{t \rightarrow+\infty} \frac{\log v^{2}}{t}=-2(N-1) .
$$

Proof of Theorem 1.2. By Lemmas 3.1 and 3.2, the proof is completed.

4. Existence of radial solutions of (1.1). Now we denote the functional

$$
\begin{aligned}
& I(z)=I(u, v)=\int_{\mathbb{\boxplus}^{N}} \nabla_{\mathbb{\boxplus}^{N}} u \cdot \nabla_{\mathbb{H}^{N}} v d V_{\mathbb{\boxplus}^{N}}-\frac{1}{p+1} \int_{\mathbb{H}^{N}}\left(v_{+}\right)^{p+1} d V_{\mathbb{U}^{N}} \\
& -\frac{1}{q+1} \int_{\mathbb{\mathbb { M } ^ { N }}}\left(u_{+}\right)^{q+1} d V_{\mathbb{\llbracket}},
\end{aligned}
$$

which is the functional of problem (1.1), where $z=(u, v), u_{+}=\max \{u, 0\}, v_{+}=$ $\max \{v, 0\}$.

Observe that the quadratic part of $I$ is well defined if $u, v \in H^{1}\left(\mathbb{H}^{N}\right)$. But if we take such $u$ and $v$, then nonlinear part is well defined if $p$ and $q$ are less or equal to $\frac{N+2}{N-2}$, for $N \geq 3$. However, we would like to consider pairs $(p, q)$ that do not satisfy this restriction. The basic requirement would be that $(p, q)$ is below the critical hyperbola, one of them could be larger than $\frac{N+2}{N-2}$. So we need inhomogeneous Sobolev spaces on hyperbolic space.

Now we define the inhomogeneous Sobolev space on $\mathbb{H}^{N}$. Let $L_{r}^{2}\left(\mathbb{H}^{N}\right)$ be the space of $L^{2}$-functions in $\mathbb{H}^{N}$ which are radially symmetric, and $T=-\Delta_{\mathbb{\boxplus}^{N}}$ with the domain $D(T)=H_{r}^{2}\left(\mathbb{M}^{N}\right)$ which is the space of radial symmetric functions that are in $L^{2}$ and 
have second derivatives in $L^{2}$. For $0 \leq s \leq 2$, the space $E^{s}$, which is the domain $D\left(T^{\frac{s}{2}}\right)$, is precisely the space obtained by interpolation between $H_{r}^{2}\left(\mathbb{M}^{N}\right)$ and $L_{r}^{2}\left(\mathbb{M}^{N}\right)$,

$$
\left[H_{r}^{2}\left(\mathbb{M}^{N}\right), L_{r}^{2}\left(\mathbb{M}^{N}\right)\right]_{1-\frac{s}{2}} .
$$

In this case, the space $E^{s}$ is the usual Sobolev space $H_{r}^{s}\left(\mathbb{H}^{N}\right)$. So denoting by $A=$ $\left(-\Delta_{\mathbb{U}^{N}}\right)^{\frac{1}{2}}$, we have for all $0 \leq s \leq 2$,

$$
D\left(A^{s}\right)=H_{r}^{s}\left(\mathbb{\boxplus}^{N}\right) .
$$

Moreover, we have $H_{r}^{s} \hookrightarrow H_{r}^{t}$ for $s>t$ (see [27]), and the Sobolev embedding theorem

$$
H_{r}^{s} \hookrightarrow L^{q}, \text { if } 2 \leq q<\infty, \text { and } s=\frac{N}{2}-\frac{N}{q}
$$

holds.

Now we have the following imbedding theorem. The case $s=1$ was proved by [5].

LEMMA 4.1. Let $s>0$, then the restriction to $H_{r}^{s}\left(\mathbb{M}^{N}\right)$ of the Sobolev imbedding of $H^{s}\left(\mathbb{H}^{N}\right)$ into $L^{q}\left(\mathbb{H}^{N}\right)$ is continuous if $2 \leq q \leq \frac{2 N}{N-2 s}$, and it is compact if $2<q<\frac{2 N}{N-2 s}$.

Proof.

Case 1. When $s>1$.

By the definition of $H^{s}\left(\mathbb{H}^{N}\right)$, we have

$$
H_{r}^{s}\left(\dddot{M}^{N}\right) \hookrightarrow H_{r}^{1}\left(\mathbb{H}^{N}\right) .
$$

So, if $u \in H_{r}^{s}\left(\mathbb{M}^{N}\right)$, we have that $u \in L^{q}\left(\mathbb{M}^{N}\right), 2 \leq q \leq \frac{2 N}{N-2}$ and $u \in L^{\frac{2 N}{N-2 s}}\left(\mathbb{M}^{N}\right)$. For $\frac{2 N}{N-2}<q<\frac{2 N}{N-2 s}$, by an interpolation inequality, we have

$$
\|u\|_{L^{q}\left(\mathbb{M}^{N}\right)} \leq\|u\|_{L^{\frac{2 N}{N-2}\left(\mathbb{A}^{N}\right)}}^{\theta}\|u\|_{L^{\frac{2 N}{N-2 s}\left(\mathbb{N}^{N}\right)}}^{1-\theta},
$$

where $\frac{1}{q}=\frac{\theta(N-2)}{2 N}+\frac{(1-\theta)(N-2 s)}{2 N}$. This shows that the map $i: H_{r}^{s}\left(\mathbb{H}^{N}\right) \rightarrow L^{q}\left(\mathbb{H}^{N}\right)$ for all $2 \leq q \leq \frac{2 N}{N-2 s}$ is continuous.

Now we will show that the map $i: H_{r}^{s}\left(\mathbb{M}^{N}\right) \rightarrow L^{q}\left(\mathbb{M}^{N}\right)$ for all $2<q<\frac{2 N}{N-2 s}$ is compact. From [5], we have that $H_{r}^{1}\left(\mathbb{H}^{N}\right) \hookrightarrow L^{p}\left(\mathbb{H}^{N}\right), 2<p<\frac{2 N}{N-2}$ is compact. By the definition of $H^{s}\left(\mathbb{M}^{N}\right)$, we also have

$$
H_{r}^{s}\left(\mathbb{H}^{N}\right) \hookrightarrow H_{r}^{1}\left(\mathbb{M}^{N}\right) .
$$

Hence,

$$
H_{r}^{s}\left(\mathbb{M}^{N}\right) \hookrightarrow L^{q}\left(\mathbb{W}^{N}\right), 2<q<\frac{2 N}{N-2} \text { is compact. }
$$

If $\frac{2 N}{N-2} \leq q<\frac{2 N}{N-2 s}$, we can deduce this lemma by using Sobolev inequalities and Hölder inequalities. Indeed, for $a \in(0,1)$ and $2<p<\frac{2 N}{N-2}$, we get

$$
\begin{aligned}
& \int_{\mathbb{\boxplus}^{N}}|u|^{q} d V_{\mathbb{\boxplus}^{N}}=\int_{\mathbb{G}^{N}}|u|^{q-p a}|u|^{p a} d V_{\mathbb{\boxplus}^{N}} \\
& \leq\left(\int_{\mathbb{M}^{N}}|u|^{p} d V_{\mathbb{\boxplus}^{N}}\right)^{a}\left(\int_{\mathbb{\mathbb { W } ^ { N }}}|u|^{\frac{q-p a}{1-a}} d V_{\mathbb{\boxplus}^{N}}\right)^{1-a} .
\end{aligned}
$$


Now we only need to check that

$$
\frac{q-p a}{1-a} \leq \frac{2 N}{N-2 s}
$$

It is easy to check that (4.1) holds if and only if

$$
q(N-2 s) \leq 2 N(1-a)+p a(N-2 s)
$$

holds. Thus, for a fixed $q<\frac{2 N}{N-2 s}$, (4.2) may easily be achieved by choosing ' $a$ ' as sufficiently small.

Case 2. When $0<s<1$.

From [27], we have that

$$
\left[L^{2}\left(\mathbb{M}^{N}\right), H^{1}\left(\mathbb{M}^{N}\right)\right]_{s}=H^{s}\left(\mathbb{M}^{N}\right),
$$

and

$$
\left[L_{r}^{2}\left(\mathbb{W}^{N}\right), H_{r}^{1}\left(\mathbb{H}^{N}\right)\right]_{s}=H_{r}^{s}\left(\mathbb{M}^{N}\right) .
$$

From Section 2, setting $|\xi|=\tanh \frac{t}{2}, u(t)=u\left(\tanh \frac{t}{2}\right), k(t)=(\sinh t)^{N-1}$, we have

$$
\begin{aligned}
& \int_{\mathbb{\mathbb { H } ^ { N }}}|u|^{q} d V_{\mathbb{T}^{N}}=w_{N-1} \int_{0}^{\infty} k(t)|u|^{q} d t, \\
& \int_{\mathbb{\boxplus}^{N}}\left|\nabla_{\mathbb{\boxplus}^{N}} u\right|^{2} d V_{\mathbb{\boxplus}^{N}}=w_{N-1} \int_{0}^{\infty} k(t)\left|u^{\prime}\right|^{2} d t .
\end{aligned}
$$

Now we claim that:

$$
\left\|(\sinh t)^{\frac{N-1}{2}} u(t)\right\|_{H^{1}(1, \infty)} \leq C\|u\|_{H^{1}\left(\mathbb{M}^{N}\right)}, \quad \forall u \in H_{r}^{1}\left(\mathbb{M}^{N}\right) .
$$

To do this, let $v(t)=(\sinh t)^{\frac{N-1}{2}} u(t)$, we have

$$
\begin{aligned}
\left|\frac{d v}{d t}\right| & =\left|\frac{N-1}{2}(\sinh t)^{\frac{N-3}{2}}(\cosh t) u(t)+(\sinh t)^{\frac{N-1}{2}} u^{\prime}(t)\right| \\
& \leq C \frac{(\sinh t)^{\frac{N-1}{2}}}{\tanh t}|u|+(\sinh t)^{\frac{N-1}{2}}\left|\frac{d u}{d t}\right| .
\end{aligned}
$$

It implies that

$$
\begin{aligned}
\int_{1}^{\infty}\left|\frac{d v}{d t}\right|^{2} d t & \leq C\left(\int_{0}^{\infty}(\sinh t)^{N-1} u^{2}(t) d t+\int_{0}^{\infty}(\sinh t)^{N-1}\left|\frac{d u}{d t}\right|^{2} d t\right) \\
& \leq C\|u\|_{H^{1}\left(\mathbb{H}^{N}\right) .}
\end{aligned}
$$

For

$$
\int_{0}^{\infty}(\sinh t)^{N-1} u^{2}(t) d t=w_{N-1}^{-1}\|u\|_{L^{2}(\mathbb{M} N)},
$$

and by interpolation, we get

$$
\left\|(\sinh t)^{\frac{N-1}{2}} u(t)\right\|_{H^{s}(1, \infty)} \leq C\|u\|_{H_{r}^{s}\left(\mathbb{\boxplus} \mathbb{N}^{N}\right)} .
$$


Thus, we can easily deduce this lemma by $\forall q \in\left(2, \frac{2 N}{N-2 s}\right)$,

$$
\begin{gathered}
\|u\|_{L^{q}\left(\mathbb{M}^{N}\right)} \leq C\|u\|_{H^{s}\left(\mathbb{\boxplus}^{N}\right)}, \quad \forall u \in H_{r}^{s}\left(\mathbb{\boxplus}^{N}\right), \\
\left\|(\sinh t)^{\frac{N-1}{2}} u(t)\right\|_{L^{q}(1, \infty)} \leq C\|u\|_{H^{s}\left(\mathbb{N}^{N}\right) .}
\end{gathered}
$$

Indeed, we have

$$
\begin{aligned}
\int_{R}^{\infty}(\sinh t)^{N-1}|u(t)|^{q} d t & \leq \frac{1}{\left(\frac{e^{R}+e^{-R}}{2}\right)^{(N-1)\left(\frac{q}{2}-1\right)}} \int_{R}^{\infty}(\sinh t)^{\frac{(N-1) q}{2}}|u(t)|^{q} d t \\
& \leq \frac{\mathcal{C}}{\left(\frac{e^{R}+e^{-R}}{2}\right)^{(N-1)\left(\frac{q}{2}-1\right)}}\|u\|_{H^{s}\left(\mathbb{H} \mathbb{H}^{N}\right)}
\end{aligned}
$$

It implies that if $R$ is large enough,

$$
w_{N-1} \int_{R}^{\infty}(\sinh t)^{N-1}|u(t)|^{q} d t \leq \varepsilon, \quad \forall u \in H_{r}^{s}\left(\mathbb{M}^{N}\right) .
$$

Now, let $s, t>0$ with $s+t=2$, we define the Hilbert space $E=H_{r}^{s}\left(\mathbb{H}^{N}\right) \times$ $H_{r}^{t}\left(\mathbb{W}^{N}\right)$, and the bilinear form: $B: E \times E \longrightarrow \mathbb{R}$ is defined by

$$
B[(u, v),(\varphi, \psi)]=\int_{\mathbb{H}^{N}} A^{s} u A^{t} \psi+A^{s} \varphi A^{t} v,
$$

and the corresponding quadratic form associated with the bilinear form $B$ is

$$
Q(z)=\int_{\mathbb{W}^{N}} A^{s} u A^{t} v, \quad(u, v) \in E .
$$

As in [12], we have $E=E^{-} \oplus E^{+}$and $B\left[z^{+}, z^{-}\right]=0$ for $z^{+} \in E^{+}, z^{-} \in E^{-}$, where

$$
E^{-}=\left\{\left(u,-A^{-t} A^{s} u\right): u \in H_{r}^{s}\left(\mathbb{H}^{N}\right)\right\}, \quad E^{+}=\left\{\left(u, A^{-t} A^{s} u\right): u \in H_{r}^{s}\left(\mathbb{M}^{N}\right)\right\} .
$$

We also have $Q(z)=\frac{1}{2} B[z, z]$ and $\frac{1}{2}\|z\|_{E}^{2}=Q\left(z^{+}\right)-Q\left(z^{-}\right)$, where $z=(u, v) \in E$ and $z=z^{+}+z^{-}, z^{+} \in E^{+}, z^{-} \in E^{-}$.

Now we consider the existence of solutions for problem (1.1). We choose $s, t>0$, and $s+t=2$ such that

$$
2<p+1<\frac{2 N}{N-2 t}, \quad 2<q+1<\frac{2 N}{N-2 s} .
$$

By the assumptions in Theorem 1.3, we know that such an ' $s$ ' exists.

We consider the functional $I: E \longrightarrow \mathbb{R}$, defined by

$$
I(z)=\int_{\mathbb{\boxplus}^{N}} A^{s} u A^{t} v d V_{\mathbb{\boxplus}^{N}}-\frac{1}{p+1} \int_{\mathbb{\boxplus}^{N}}\left(v_{+}\right)^{p+1} d V_{\mathbb{\boxplus}^{N}}-\frac{1}{q+1} \int_{\mathbb{\boxplus}^{N}}\left(u_{+}\right)^{q+1} d V_{\mathbb{\boxplus}^{N}}
$$


for $z=(u, v) \in E . I$ is a $C^{1}$ functional and

$$
\left\langle I^{\prime}(z), \eta\right\rangle_{E}=\int_{\mathbb{\boxplus}^{N}} A^{s} u A^{t} \psi+A^{s} \varphi A^{t} v-\int_{\mathbb{\boxplus}^{N}}\left(v_{+}\right)^{p} \psi-\int_{\mathbb{\boxplus}^{N}}\left(u_{+}\right)^{q} \varphi
$$

for $z=(u, v) \in E$ and $\eta=(\varphi, \psi) \in E$. So the critical points of $I$ satisfy the equations

$$
\int_{\mathbb{\boxplus}^{N}} A^{s} u A^{t} \psi-\int_{\mathbb{Q}^{N}}\left(v_{+}\right)^{p} \psi=0, \text { for all } \psi \in H_{r}^{t}\left(\mathbb{H}^{N}\right)
$$

and

$$
\int_{\mathbb{H}^{N}} A^{s} \varphi A^{t} v-\int_{\mathbb{U}^{N}}\left(u_{+}\right)^{q} \varphi=0, \text { for all } \varphi \in H_{r}^{s}\left(\mathbb{M}^{N}\right) .
$$

We shall use a linking theorem in [18] to find critical points of $I$. To apply the theorem, we introduce finite dimensional spaces in $E$. Let $\left\{e_{j}\right\}, j=1,2, \ldots$, be a complete orthogonal systems in $H_{r}^{s}\left(\mathbb{H}^{N}\right)$. Let $H_{n}$ denote the finite dimensional subspaces of $H_{r}^{s}\left(\mathbb{H}^{N}\right)$ generated by $\left\{e_{j}\right\}, j=1,2, \ldots, n$. As $A^{s}: H_{r}^{s}\left(\mathbb{H}^{N}\right) \rightarrow L_{r}^{2}\left(\mathbb{H}^{N}\right)$ and $A^{t}: H_{r}^{t}\left(\mathbb{W}^{N}\right) \rightarrow L_{r}^{2}\left(\mathbb{W}^{N}\right)$ are isomorphisms, we see that $\left\{\hat{e}_{j}\right\}, j=1,2, \ldots$, where $\hat{e}_{j}=A^{-t} A^{s} e_{j}$ is a complete orthogonal system in $H_{r}^{t}\left(\mathbb{H}^{N}\right)$. Let $\hat{H}_{n}$ denote the finite dimensional subspaces of $H_{r}^{t}\left(\mathbb{H}^{N}\right)$ generated by $\left\{\hat{e}_{j}\right\}, j=1,2, \ldots, n$. For each $n \in \mathbb{N}$, we introduce the following subspaces of $E^{+}$and $E^{-}$respectively:

$$
\begin{gathered}
E_{n}^{+}=\operatorname{span}\left\{\left(e_{j}, \hat{e}_{j}\right) \in E^{+}\right\}, \quad j=1,2, \ldots, n, \\
E_{n}^{-}=\operatorname{span}\left\{\left(e_{j},-\hat{e}_{j}\right) \in E^{-}\right\}, \quad j=1,2, \ldots, n .
\end{gathered}
$$

Let $E_{n}=E_{n}^{+} \oplus E_{n}^{-}$. We say that the functional $I$ satisfies the $(P S)^{*}$ condition if any sequence $\left\{z_{n}\right\}, z_{n} \in E_{n}$ such that

$$
\left|I\left(z_{n}\right)\right| \leq \text { const, } \quad\left|\left\langle\nabla_{\mathbb{H}^{N}} I\left(z_{n}\right), \eta\right\rangle\right| \leq \varepsilon_{n}\|\eta\|_{E}, \varepsilon_{n} \rightarrow 0, \eta \in E_{n}
$$

possesses a subsequence converging to a critical point of $I$. The functional of $I$ is said to have a local linking at 0 if for some $r>0$, one has

$$
I(z) \geq 0, \text { for } z \in E^{+},\|z\| \leq r
$$

and

$$
I(z) \leq 0, \text { for } z \in E^{-},\|z\| \leq r .
$$

Now the result in [20] can be stated as follows.

THEOREM 4.1. Suppose that $I \in C^{1}(E, \mathbb{R})$ satisfies the following assumptions:

(B1) I has a local linking at 0 ,

(B2) I satisfies $(P S)^{*}$,

(B3) I maps bounded sets into bounded sets,

(B4) for every $n \in \mathbb{N}, I(z) \rightarrow-\infty$ as $|z| \rightarrow \infty$ and $z \in E_{n}^{+} \oplus E^{-}$.

Then I has a non-trivial critical point.

Proof of Theorem 1.3. We shall verify that $I$ satisfies the conditions in Theorem 4.1. Condition (B3) is an immediate consequence of the continuity of the bilinear form 
$B$ and the assumption of $p, q$. Next, we will check (B1). For $z=(u, v) \in E^{+}$, we have

$$
I(z)=I(u, v) \geq c_{0}\|z\|_{E}^{2}-C\|u\|_{H_{r}^{s}\left(\mathbb{M}^{N}\right)}^{q+1}-C\|v\|_{H_{r}^{t}\left(\mathbb{H}^{N}\right)}^{p+1}
$$

for some $c_{0}>0$. And since $p, q>1$, we conclude that $I(z) \geq 0$ for $z \in E^{+}$with $\|z\|$ small. A similar argument prove that $I(z)=I(u, v) \leq 0$ for $z \in E^{-}$with $\|z\|$ small.

Now we verify (B4). Let $n \in \mathbb{N}$ be fixed and $z \in E_{n}^{+} \oplus E^{-}$, with $\|z\| \rightarrow \infty$, we claim that $I(z) \rightarrow-\infty$. Indeed, with $z=(u, v)$ and $z=z^{+}+z^{-}$, we have

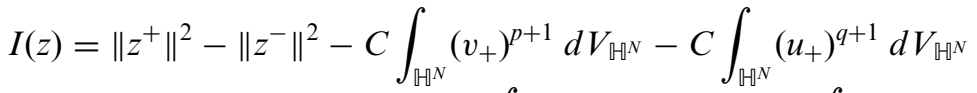

$$
\begin{aligned}
& \leq-c_{0}\left\|z^{-}\right\|_{E}^{2}+C\left\|z^{+}\right\|_{E}^{2}-C \int_{\mathbb{G}}\left(v_{+}\right)^{p+1} d V_{\mathbb{\boxplus}^{N}}-C \int_{\mathbb{W}^{N}}\left(u_{+}\right)^{q+1} d V_{\mathbb{M}^{N}} .
\end{aligned}
$$

Let $z^{+}=\left(u^{+}, v^{+}\right)$and $z^{-}=\left(u^{-}, v^{-}\right)$, we have $u^{-}=\gamma u^{+}+\hat{u}$, where $\hat{u}$ is orthogonal to $u^{+}$in $L^{2}$. Also, we have $v^{-}=\mu v^{+}+\hat{v}$, where $\hat{v}$ is orthogonal to $v^{-}$in $L^{2}$. We may verify that either $\gamma$ or $\mu$ is positive.

Suppose $\gamma>0$, then we have

$$
(1+\gamma) \int_{\mathbb{\boxplus}^{N}}\left|u^{+}\right|^{2} d V_{\mathbb{\boxplus}^{N}}=\int_{\mathbb{\mathbb { M } ^ { N }}}\left[(1+\gamma) u^{+}+\hat{u}\right] u^{+} d V_{\mathbb{\boxplus}^{N}} \leq\|u\|_{L^{q+1}}\left\|u^{+}\right\|_{L^{\frac{q+1}{q}}} .
$$

As the norms in $E_{n}^{+}$are equivalent, we obtain

$$
(1+\gamma)\left\|u^{+}\right\|_{L^{q+1}} \leq C\|u\|_{L^{q+1}},
$$

where constant $C$ is independent of $u$. So we obtain

$$
I(z) \leq-c_{0}\left\|z^{-}\right\|^{2}+C\left\|z^{+}\right\|^{2}-C\left\|u^{+}\right\|^{q+1},
$$

which implies that $I(z) \rightarrow-\infty$ as $\|z\| \rightarrow \infty$.

Finally, we verify the $(P S)^{*}$ condition. Suppose $\left\{z_{n}\right\} \subset E_{n}$ is a sequence satisfying

$$
\left|I\left(z_{n}\right)\right| \leq C, \quad\left|\left\langle\nabla_{\mathbb{\boxplus}^{N}}\left(z_{n}\right), \eta\right\rangle\right| \leq \varepsilon_{n}\|\eta\|_{E}, \text { for } \eta \in E_{n},
$$

where $\varepsilon_{n} \rightarrow 0$ as $n \rightarrow \infty$. We need only to show that $\left\|z_{n}\right\|=\left\|\left(u_{n}, v_{n}\right)\right\|$ is bounded uniformly. Indeed, if $\left\|z_{n}\right\|$ is uniformly bounded, by using Lemma 4.1 , we see that $u_{n}$ and $v_{n}$ converge strongly up to a subsequence in $L^{p+1}$ and $L^{q+1}$ respectively, the conclusion here doesn't follow in a standard way but can be proved as in [12]. Taking $\eta=z_{n}$, we have

$$
\begin{aligned}
C+\varepsilon_{n}\left\|z_{n}\right\| & \geq I\left(z_{n}\right)-\frac{1}{2}\left\langle I^{\prime}\left(z_{n}\right), z_{n}\right\rangle \\
& =\left(\frac{1}{2}-\frac{1}{p+1}\right) \int_{\mathbb{\boxplus}^{N}}\left[\left(v_{n}\right)_{+}\right]^{p+1} d V_{\mathbb{\boxplus}^{N}}+\left(\frac{1}{2}-\frac{1}{q+1}\right) \int_{\mathbb{\boxplus}^{N}}\left[\left(u_{n}\right)_{+}\right]^{q+1} d V_{\mathbb{M}^{N}} .
\end{aligned}
$$

Now we estimate $\left\|u_{n}\right\|_{H_{r}^{s}}$ and $\left\|v_{n}\right\|_{H_{r}^{t}}$. From (4.3), we have

$$
\begin{aligned}
& \left|\int_{\mathbb{\mathbb { T } ^ { N }}} A^{s} \varphi A^{t} v_{n}\right| \leq\left|\int_{\mathbb{\boxplus}^{N}}\left[\left(u_{n}\right)_{+}\right]^{q} \varphi\right|+\varepsilon_{n}\|\varphi\|_{H_{r}^{s}} \\
& \leq\left[\left(\int_{\mathbb{\boxplus}^{N}}\left[\left(u_{n}\right)_{+}\right]^{q+1} d V_{\mathbb{\boxplus}^{N}}\right)^{\frac{q}{q+1}}+\varepsilon_{n}\right]\|\varphi\|_{H_{r}^{s}}, \text { for all } \varphi \in H_{n},
\end{aligned}
$$


which implies that

$$
\left\|v_{n}\right\|_{H_{r}^{t}} \leq\left(\int_{\mathbb{W}^{N}}\left[\left(u_{n}\right)_{+}\right]^{q+1} d V_{\mathbb{\mathbb { H } ^ { N }}}\right)^{\frac{q}{q+1}}+\varepsilon_{n}
$$

Similarly, we prove that

$$
\left\|u_{n}\right\|_{H_{r}^{s}} \leq\left(\int_{\mathbb{\mathbb { N } ^ { N }}}\left[\left(v_{n}\right)_{+}\right]^{p+1} d V_{\mathbb{\mathbb { Q } ^ { N }}}\right)^{\frac{p}{p+1}}+\varepsilon_{n}
$$

Thus, we conclude that

$$
\left\|u_{n}\right\|_{H_{r}^{s}}+\left\|v_{n}\right\|_{H_{r}^{t}} \leq\left(\int_{\mathbb{\boxplus}^{N}}\left[\left(v_{n}\right)_{+}\right]^{p+1} d V_{\mathbb{G}^{N}}\right)^{\frac{p}{p+1}}+\left(\int_{\mathbb{H}^{N}}\left[\left(u_{n}\right)_{+}\right]^{q+1} d V_{\mathbb{Q}^{N}}\right)^{\frac{q}{q+1}}+2 \varepsilon_{n} .
$$

Using this estimate in (4.4), we get

$$
\left\|\left(u_{n}\right)_{+}\right\|_{L^{q+1}}^{q+1}+\left\|\left(v_{n}\right)_{+}\right\|_{L^{p+1}}^{p+1} \leq C\left(\left\|\left(u_{n}\right)_{+}\right\|_{L^{q+1}}^{q}+\left\|\left(v_{n}\right)_{+}\right\|_{L^{p+1}}^{p}\right)+C .
$$

Hence, both $\left\|\left(u_{n}\right)_{+}\right\|_{L^{q+1}}$ and $\left\|\left(v_{n}\right)_{+}\right\|_{L^{p+1}}$ are bounded, and consequently $\left\|u_{n}\right\|_{H_{r}^{s}}$ and $\left\|v_{n}\right\|_{H_{r}^{t}}$ are also bounded in view of (4.5). The proof of Theorem 1.3 is completed.

Now we consider the existence of a radial positive ground state for problem (1.1). For $p, q<\frac{N+2}{N-2}$, then $\frac{1}{p+1}+\frac{1}{q+1}>\frac{N-2}{N}$. By Theorem 1.3, we know that the set

$$
\Lambda=\left\{(u, v) \in H_{r}^{1}\left(\mathbb{M}^{N}\right) \times H_{r}^{1}\left(\mathbb{H}^{N}\right): I^{\prime}(u, v)=0,(u, v) \not \equiv(0,0)\right\},
$$

is a non-empty set. We defined a minimization problem

$$
I^{\infty}=\inf \{I(u, v) \mid(u, v) \in \Lambda\}
$$

We recall that if a critical point of $I$ achieved $I^{\infty}$ in (4.6), it is called a ground state for problem (1.1), see [4].

The proof of Theorem 1.4 will be completed by the following proposition.

Proposition 4.1. If $1<p, q<\frac{N+2}{N-2}$, then $I^{\infty}$ is attained and $I^{\infty}>0$.

Proof. If $p, q<\frac{N+2}{N-2}$, then $\frac{1}{p+1}+\frac{1}{q+1}>\frac{N-2}{N}$. By Theorem 1.3, there exists a nontrivial radial solution pair $(U, V) \in H_{r}^{1}\left(\mathbb{M}^{N}\right) \times H_{r}^{1}\left(\mathbb{M}^{N}\right)$ of $(1.1)$. So the set $\Lambda \neq \emptyset$ and $I^{\infty}$ are finite. If $z=(u, v)$ is a solution of $(1.1)$, then

$$
2 \int_{\mathbb{\mathbb { N } ^ { N }}} \nabla_{\mathbb{U}^{N}} u \cdot \nabla_{\mathbb{H}^{N}} v d V_{\mathbb{G}^{N}}=\int_{\mathbb{W}^{N}}\left(u_{+}\right)^{q+1}+\left(v_{+}\right)^{p+1} d V_{\mathbb{\mathbb { M } ^ { N }}},
$$


and hence,

$$
\begin{aligned}
& I(z)=I(u, v) \\
& =\int_{\mathbb{H}^{N}} \nabla_{\mathbb{U}^{N}} u \cdot \nabla_{\mathbb{U}^{N}} v d V_{\mathbb{\boxplus}^{N}}-\frac{1}{p+1} \int_{\mathbb{H}^{N}}\left(v_{+}\right)^{p+1} d V_{\mathbb{U}^{N}}-\frac{1}{q+1} \int_{\mathbb{U}^{N}}\left(u_{+}\right)^{q+1} d V_{\mathbb{U}^{N}} \\
& =\left(\frac{1}{2}-\frac{1}{p+1}\right) \int_{\mathbb{\mathbb { H } ^ { N }}}\left(v_{+}\right)^{p+1} d V_{\mathbb{W}^{N}}+\left(\frac{1}{2}-\frac{1}{q+1}\right) \int_{\mathbb{\boxplus}^{N}}\left(u_{+}\right)^{q+1} d V_{\mathbb{G}^{N}} \\
& >0 \text {. }
\end{aligned}
$$

Now, we show that $I^{\infty}$ is obtained and positive. We show that the set of non-trivial solutions is bounded from below. In fact, we have

$$
\begin{aligned}
& \left\|(u)_{+}\right\|_{H^{1}\left(\mathbb{M}^{N}\right)}^{2}=\int_{\mathbb{H}^{N}}\left(v_{+}\right)^{p} u_{+} d V_{\mathbb{\boxplus}^{N}}
\end{aligned}
$$

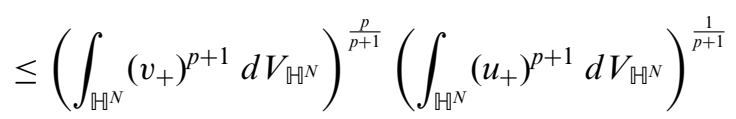

$$
\begin{aligned}
& \leq C\left\|v_{+}\right\|_{L^{p+1}\left(\mathbb{M} \mathbb{N}^{N}\right)}^{p}\left\|u_{+}\right\|_{H^{1}\left(\mathbb{M}^{N}\right)} .
\end{aligned}
$$

It implies that

$$
\|u\|_{H_{r}^{1}\left(\mathbb{M}^{N}\right)} \leq C\|v\|_{L_{r}^{p+1}\left(\mathbb{M}^{N}\right)}^{p}
$$

Now, using the two equations, we obtain

$$
\int_{\mathbb{\boxplus}^{N}}\left(v_{+}\right)^{p+1} d V_{\mathbb{\mathbb { N } ^ { N }}}=\int_{\mathbb{H}^{\mathbb{N}}} \nabla_{\mathbb{U}^{\mathbb{N}}} u \cdot \nabla_{\mathbb{H}^{N}} v d V_{\mathbb{\boxplus}^{N}}=\int_{\mathbb{H}^{N}}\left(u_{+}\right)^{q+1} d V_{\mathbb{\boxplus}^{N}} .
$$

From (4.7) and (4.8), it follows that

$$
\left\|u_{+}\right\|_{L_{r}^{q+1}\left(\mathbb{M}^{N}\right)} \leq\left\|u_{+}\right\|_{H_{r}^{1}\left(\mathbb{H}^{N}\right)} \leq C\left\|v_{+}\right\|_{L_{r}^{p+1}\left(\mathbb{H}^{N}\right)}^{p} \leq C\left\|u_{+}\right\|_{L_{r}^{q+1}\left(\mathbb{H}^{N}\right)}^{\frac{p(q+1)}{p+1}} .
$$

Thus, we get

$$
\left\|u_{+}\right\|_{L_{r}^{q+1}\left(\mathbb{M}^{N}\right)} \leq C\left\|u_{+}\right\|_{L_{r}^{q+1}\left(\mathbb{Q}^{N}\right)}^{\frac{p(q+1)}{p+1}},
$$

which implies $\left\|u_{+}\right\|_{L_{r}^{q+1}\left(\mathbb{M}^{N}\right)} \geq C$, since $\frac{p(q+1)}{p+1}>1$. Similarly, we prove that $\left\|v_{+}\right\|_{L_{r}^{p+1}\left(\mathbb{M}^{N}\right)} \geq$ C.

Finally, we observe that $I^{\infty}$ is obtained. Indeed, suppose now that $z_{n}=\left(u_{n}, v_{n}\right)$ is a minimizing sequence of $I^{\infty}$, that is

$$
I\left(z_{n}\right) \longrightarrow I^{\infty}, I^{\prime}\left(z_{n}\right)=0, z_{n} \neq 0 .
$$

Clearly, $\left\{z_{n}\right\}$ is a $(P S)_{I^{\infty}}$ sequence for $I$, therefore it follows from the last part of the proof of Theorem 1.3 that $\left(u_{n}, v_{n}\right)$ converges strongly to $(u, v)$, which is a solution of (1.1). 
To show that $I^{\infty}>0$, we note that if $z=(u, v) \neq(0,0)$ with $I^{\prime}(z) \neq 0$, then

$$
I(z)=\left(\frac{1}{2}-\frac{1}{p+1}\right) \int_{\mathbb{\boxplus}^{N}}\left(v_{+}\right)^{p+1} d V_{\mathbb{M}^{N}}+\left(\frac{1}{2}-\frac{1}{q+1}\right) \int_{\mathbb{\boxplus}^{N}}\left(u_{+}\right)^{q+1} d V_{\mathbb{M}^{N}}>0 .
$$

Then we know that there exists a non-trivial solution $z=(u, v)$ of problem (1.1) with $I(z)=I^{\infty}$. Moreover, we have $z=(u, v) \geq(0,0)$. Otherwise, we may assume that $u$ changes sign, $u=u_{+}-u_{-}, u_{+} \not \equiv 0, u_{-} \not \equiv 0, I^{\prime}(z)=0$ satisfying

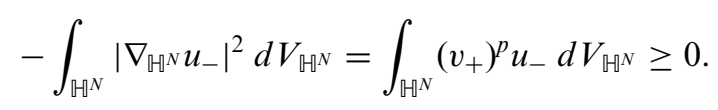

So we have that $u_{-}=0$, that is $u \geq 0$. Similarly, we have $v \geq 0$. Using the maximum principle, we obtain that $(u, v)>(0,0)$.

5. Existence of solutions in $H^{1}\left(\mathbb{M}^{N}\right) \times H^{1}\left(\mathbb{W}^{N}\right)$. Let $E=H^{1}\left(\mathbb{M}^{N}\right) \times H^{1}\left(\mathbb{M}^{N}\right)$ and the natural inner product on $E$ is given by

$$
\langle(u, v),(\varphi, \psi)\rangle_{E}=\int_{\mathbb{\boxplus}^{N}} \nabla_{\mathbb{U}^{N}} u \nabla_{\mathbb{\boxplus}^{N}} \varphi+\nabla_{\mathbb{\boxplus}^{N}} v \nabla_{\mathbb{\boxplus}^{N}} \psi d V_{\mathbb{\boxplus}^{N}},
$$

then $E$ is a separable Hilbert space with dual $E^{*}$.

Following [21], we have the direct decomposition $E=E^{+} \oplus E^{-}$, where both $E^{+}, E^{-}$are infinite dimensional, and

$$
E^{+}=\left\{(u, u), u \in H^{1}\left(\mathbb{M}^{N}\right)\right\}, \quad E^{-}=\left\{(u,-u), u \in H^{1}\left(\mathbb{M}^{N}\right)\right\} .
$$

We also see that for $z=(u, v), z=z^{+}+z^{-}$with $z^{+}=\left(\frac{u+v}{2}, \frac{u+v}{2}\right), z^{-}=\left(\frac{u-v}{2},-\frac{u-v}{2}\right)$ and

$$
\int_{\mathbb{\boxplus}^{N}} \nabla_{\mathbb{\boxplus}^{N}} u \nabla_{\mathbb{\boxplus}^{N}} v d V_{\mathbb{\boxplus}^{N}}=\frac{1}{2}\left(\left\|z^{+}\right\|^{2}-\left\|z^{-}\right\|^{2}\right),
$$

where $\left\|z^{ \pm}\right\|^{2}=2 \int_{\mathbb{U}^{N}}\left|\nabla_{\mathbb{\boxplus}^{N}}\left(\frac{u+v}{2}\right)\right|^{2} d V_{\mathbb{\boxplus}^{N}}$.

Lemma 5.1. Let $N_{r}=\left\{z \in E^{+},\|z\|=r\right\}$. Then there exists $r>0$ such that

$$
b=\inf _{N_{r}} I(z)>0 .
$$

Proof. For $z \in N_{r}$, there is a $u \in H^{1}\left(\mathbb{H}^{N}\right)$ with $z=(u, u)$. If $\|z\|$ is small, we have

$$
\begin{aligned}
& I(z)=\int_{\mathbb{\boxplus}^{N}}\left|\nabla_{\mathbb{U}^{N}} u\right|^{2} d V_{\mathbb{\boxplus}^{N}}-\frac{1}{p+1} \int_{\mathbb{M}^{N}}\left(v_{+}\right)^{p+1} d V_{\mathbb{\boxplus}^{N}}-\frac{1}{q+1} \int_{\mathbb{\boxplus}^{N}}\left(u_{+}\right)^{q+1} d V_{\mathbb{\boxplus}^{N}} \\
& \geq \frac{1}{2}\|z\|^{2}-c\|z\|^{p+1}-c\|z\|^{q+1} \geq b>0
\end{aligned}
$$

for some $b>0$.

Let $M_{R}=\left\{z=z^{-}+s z_{0}: z^{-} \in E^{-},\|z\| \leq R, s \geq 0\right\}$ for some $z_{0} \in E^{+} \backslash\{0\}$ with $\left\|z_{0}\right\|=1$.

LEMMA 5.2. There exists $R>r$ such that $(i) \max _{\partial M_{R}} I=0$; (ii) $S:=\sup _{\bar{M}_{R}} I<\infty$. 
Proof. If $z \in \partial M_{R}$, then $z=z^{-}+s z_{0}$ with either $\|z\|=R, s \geq 0$, or $\|z\|<R, s=0$. If $s=0$, we have $z \in E^{-}, z=(u,-u)$ and

$$
I(u,-u)=-\frac{1}{2}\left\|z^{-}\right\|^{2}-\frac{1}{p+1} \int_{\mathbb{G} \mathbb{W}^{N}}\left[(-u)_{+}\right]^{p+1} d V_{\mathbb{\boxplus} \mathbb{N}^{N}}-\frac{1}{q+1} \int_{\mathbb{\boxplus}^{N}}\left(u_{+}\right)^{q+1} d V_{\mathbb{\boxplus}^{N}} \leq 0 .
$$

Suppose now that $s>0,\|z\|=R$. We fix $z_{0}=\left(u_{0}, u_{0}\right), z^{-}=(u,-u)$, then $z=z^{-}+$ $s z_{0}=\left(u+s u_{0},-u+s u_{0}\right)$ and

$$
\begin{aligned}
I(z) & =\frac{1}{2} s^{2}-\frac{1}{2}\left\|z^{-}\right\|^{2}-\frac{1}{p+1} \int_{\mathbb{H}^{N}}\left[\left(s u_{0}-u\right)_{+}\right]^{p+1} d V_{\mathbb{H}^{N}}-\frac{1}{q+1} \int_{\mathbb{H}^{N}}\left[\left(s u_{0}+u\right)_{+}\right]^{q+1} d V_{\mathbb{H}^{N}} \\
& \leq \frac{1}{2} s^{2}-\frac{1}{2}\left\|z^{-}\right\|^{2}-C s^{p+1} .
\end{aligned}
$$

It follows that

$$
I(z)=I\left(z^{-}+s z_{0}\right) \rightarrow-\infty
$$

as $\left\|z^{+}+s z_{0}\right\|=R \rightarrow \infty$. Since $I(0)=0,(i)$ is satisfied for sufficiently large $R>r$.

(ii) The boundedness of $\bar{M}_{R}$ implies that $S:=\sup I<\infty$.

A sequence $\left\{z_{n}\right\} \subset E$ is called the Palais-Smale sequence $\left((P S)_{c}\right.$ sequence for short $)$ of a $C^{1}$ functional $I$ in $E$ at level $c$ if $I\left(z_{n}\right) \rightarrow c$ and $I^{\prime}\left(z_{n}\right) \rightarrow 0$ in $E^{*}$ as $n \rightarrow \infty$. In order to give the proof of Theorem 1.5, let us define the following.

Definition 5.1. For $r>0$, define $S_{r}=\left\{x \in \mathbb{R}^{N}:|x|^{2}=1+r^{2}\right\}$ and for $a \in S_{r}$ define

$$
A(a, r)=B(a, r) \cap \mathfrak{H}^{N},
$$

where $B(a, r)$ is the open ball in the Euclidean space with centre $a$ and radius $r>0$. Moreover, for the choice of $a$ and $r, \partial B(a, r)$ is orthogonal to $S^{N-1}$.

Similarly as [5], we have the following.

LEMMA 5.3. Let $r_{1}>0, r_{2}>0$ and $A\left(a_{i}, r_{i}\right), i=1,2$ be as in the above definition, then there exists $\tau \in \mathcal{I}\left(\mathbb{H}^{N}\right)$ such that $\tau\left(A\left(a_{1}, r_{1}\right)\right)=A\left(a_{2}, r_{2}\right)$, where $\mathcal{I}\left(\mathbb{H}^{N}\right)$ is the isometry group of $\mathbb{\boxplus}^{N}$.

The proof of the next lemma closely follows the proof of Theorem 3.3 in [5].

LEMMA 5.4. Problem (1.1) possesses at least one positive solution pair that belongs to $H^{1}\left(\mathbb{W}^{N}\right) \times H^{1}\left(\mathbb{M}^{N}\right)$.

Proof. By Lemma 5.1, there exist $r>0$ and $b>0$ such that $\left.I\right|_{N_{r}} \geq b$, where $N_{r}=$ $\left\{z \in E^{+}:\|z\|=r\right\}$. By Lemma 5.2, for such an $r$, there exist $R>r$ and suitable $z_{0} \in$ $E^{+} \backslash\{0\}$ such that $\left.I\right|_{\partial M_{R}} \leq 0$, where $M_{R}$ was given before Lemma 5.2. As $E=E^{+} \oplus E^{-}$ and for $z=(u, v) \in E$, we have

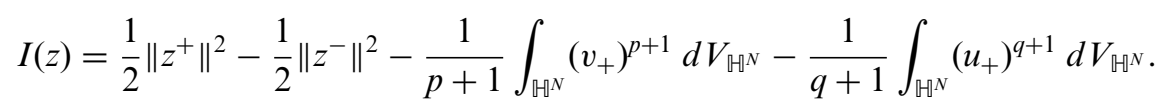

We also see that

$$
\psi(u, v):=\frac{1}{p+1} \int_{\mathbb{\boxplus}^{N}}\left(v_{+}\right)^{p+1} d V_{\mathbb{\boxplus ^ { N }}}-\frac{1}{q+1} \int_{\mathbb{\boxplus}^{N}}\left(u_{+}\right)^{q+1} d V_{\mathbb{\boxplus}^{N}}+\frac{1}{q+1} \int_{\mathbb{\boxplus}^{N}}\left(u_{+}\right)^{q+1} d V_{\mathbb{\boxplus}^{N}}
$$


is $C^{1}$, and $\psi \geq 0$ is weakly sequentially lower semi-continuous by the fact that $H^{1}\left(\mathbb{M}^{N}\right) \times H^{1}\left(\mathbb{M}^{N}\right) \subset L_{\mathrm{loc}}^{q}\left(\mathbb{M}^{N}\right) \times L_{\mathrm{loc}}^{q}\left(\mathbb{H}^{N}\right)$ and Fatou's Lemma, and $\psi^{\prime}$ is weakly sequentially continuous in the dual space of $H^{1}\left(\mathbb{H}^{N}\right) \times H^{1}\left(\mathbb{H}^{N}\right)$. Thus, there is a $(P S)_{c}$ sequence $z_{n}$ for $I$, where $I\left(z_{n}\right) \rightarrow c \geq b$. Let $z_{n}=\left(u_{n}, v_{n}\right)$, we know that there is $C<+\infty$ such that $\left\|z_{n}\right\| \leq C$ for any $n$. We may assume that

$$
z_{n} \rightarrow z \text { in } E \text {, as } n \rightarrow \infty .
$$

We claim that there exists $\delta>0$,

$$
\liminf _{n \rightarrow \infty} \int_{\mathbb{\boxplus}^{N}}\left[\left(v_{n}\right)_{+}\right]^{p+1} d V_{\mathbb{\boxplus}^{N}}=\liminf _{n \rightarrow \infty} \int_{\mathbb{M}^{N}}\left[\left(u_{n}\right)_{+}\right]^{q+1} d V_{\mathbb{M}^{N}}>\delta>0 .
$$

Since $\left\{z_{n}\right\}$ is a PS sequence, we have

$$
o(1)+\int_{\mathbb{M}^{N}}\left[\left(v_{n}\right)_{+}\right]^{p+1} d V_{\mathbb{\boxplus}^{N}}=\int_{\mathbb{U}^{N}} \nabla_{\mathbb{H}^{N}} u_{n} \cdot \nabla_{\mathbb{U}^{N}} v_{n} d V_{\mathbb{\boxplus}^{N}}=\int_{\mathbb{\boxplus}^{N}}\left[\left(u_{n}\right)_{+}\right]^{q+1} d V_{\mathbb{\boxplus}^{N}}+o(1) .
$$

If $\lim _{n \rightarrow \infty} \int_{\llbracket^{N}}\left[\left(v_{n}\right)_{+}\right]^{p+1} d V_{\llbracket^{N}}=\lim _{n \rightarrow \infty} \int_{\mathbb{\boxplus}^{N}}\left[\left(u_{n}\right)_{+}\right]^{q+1} d V_{\llbracket \square^{N}} \rightarrow 0$, then we have

$$
\int_{\mathbb{H}^{\mathbb{N}}} \nabla_{\mathbb{\mathbb { A } ^ { N }}} u_{n} \cdot \nabla_{\mathbb{\mathbb { H } ^ { N }}} v_{n} d V_{\mathbb{U}^{N}} \rightarrow 0 \text { as } n \rightarrow \infty .
$$

Thus, $I\left(z_{n}\right) \rightarrow 0$ as $n \rightarrow \infty$. This contradicts $I\left(z_{n}\right) \rightarrow c \geq b>0$ as $n \rightarrow \infty$. The claim follows.

Let us fix $\delta_{1}>0, \delta_{2}>0$ such that $0<\delta_{1}<\delta<S_{N, p}^{2 \frac{p+1}{p-1}}, 0<\delta_{2}<\delta<S_{N, p}^{2 \frac{q+1}{q-1}}$, where $S_{N, P}$ satisfies that

$$
S_{N, p}\left(\int_{\mathbb{\boxplus}^{N}}|u|^{p+1} d V_{\mathbb{\boxplus}^{N}}\right)^{\frac{2}{p+1}} \leq \int_{\mathbb{\boxplus}^{N}}\left|\nabla_{\mathbb{\boxplus}^{N}} u\right|^{2} d V_{\mathbb{U}^{N}}, \forall u \in H^{1}\left(\mathbb{\boxplus}^{N}\right), 1<p<\frac{N+2}{N-2}, N \geq 3 .
$$

Let us define the concentration function: $Q_{n}:(0, \infty) \longrightarrow \mathbb{R}$ as follows:

$$
Q_{n}(r)=\sup _{x \in S_{r}} \int_{A(x, r)}\left[\left(u_{n}\right)_{+}\right]^{q+1} d V_{\mathbb{Q}^{N}}
$$

Now $\lim _{r \rightarrow 0} Q_{n}(r)=0$, and $\lim _{r \rightarrow \infty} Q_{n}(r)>\delta_{2}$ for large $r . A(x, r)$ approximates the intersection of $\mathbb{H}^{N}$ with a half space $\left\{y \in \mathbb{R}^{N}:(y, x)>0\right\}$. Therefore, we can choose a sequence $R_{n}>0$ and $x_{n} \in S_{R_{n}}$ such that

$$
\sup _{x \in S_{R_{n}}} \int_{A\left(x, R_{n}\right)}\left[\left(u_{n}\right)_{+}\right]^{q+1} d V_{\llbracket \boxplus^{N}}=\int_{A\left(x_{n}, R_{n}\right)}\left[\left(u_{n}\right)_{+}\right]^{q+1} d V_{\mathbb{⿴ 囗 十}}=\delta_{2} .
$$

For $x_{0} \in S_{\sqrt{3}}$, and using Lemma 5.3, choosing $T_{n} \in I\left(\mathbb{H}^{N}\right)$ such that

$$
A\left(x_{n}, R_{n}\right)=T_{n}\left(A\left(x_{0}, \sqrt{3}\right)\right) .
$$

Now define $z_{n}^{\prime}=\left(u_{n}^{\prime}, v_{n}^{\prime}\right)=\left(u_{n} \circ T_{n}(x), v_{n} \circ T_{n}(x)\right)$. Since $T_{n}$ is an isometry, one can easily see that $\left\{\left(u_{n}^{\prime}, v_{n}^{\prime}\right)\right\}$ is a $(P S)_{c}$ sequence of $I$ at the same level $I^{\infty}$ as $\left(u_{n}, v_{n}\right)$, and

$$
\int_{\mathbb{\boxplus}^{N}}\left[\left(u_{n}^{\prime}\right)_{+}\right]^{q+1} d V_{\mathbb{\boxplus}^{N}}=\int_{\mathbb{\boxplus}^{N}}\left[\left(v_{n}^{\prime}\right)_{+}\right]^{p+1} d V_{\mathbb{\boxplus}^{N}}+o(1) .
$$


We also have

$$
\begin{aligned}
& \int_{A\left(x_{0}, \sqrt{3}\right)}\left[\left(u_{n}^{\prime}\right)_{+}\right]^{q+1} d V_{\mathbb{\boxplus}^{N}}=\int_{A\left(x_{n}, R_{n}\right)}\left[\left(u_{n}\right)_{+}\right]^{q+1} d V_{\mathbb{M}^{N}}=\sup _{x \in S_{\sqrt{3}}} \int_{A(x, \sqrt{3})}\left[\left(u_{n}^{\prime}\right)_{+}\right]^{q+1} d V_{\mathbb{\boxplus}}=\delta_{2}, \\
& \int_{A\left(x_{0}, \sqrt{3}\right)}\left[\left(v_{n}^{\prime}\right)_{+}\right]^{p+1} d V_{\mathbb{\boxplus}^{N}}=\int_{A\left(x_{n}, R_{n}\right)} \mid\left[\left(v_{n}\right)_{+}\right]^{p+1} d V_{\mathbb{\boxplus}^{N}} \leq \sup _{x \in S_{r}} \int_{A(x, \sqrt{3})}\left[\left(v_{n}^{\prime}\right)_{+}\right]^{p+1} d V_{\mathbb{\boxplus}^{N}}=\delta_{1},
\end{aligned}
$$

and $\left\|z_{n}\right\|=\left\|z_{n}^{\prime}\right\|$. Therefore, up to a subsequence, we may assume

$$
\begin{gathered}
z_{n}^{\prime} \rightarrow z^{\prime}=\left(u^{\prime}, v^{\prime}\right), \text { in } H^{1}\left(\mathbb{M}^{N}\right) \times H^{1}\left(\mathbb{H}^{N}\right), \\
z_{n}^{\prime} \longrightarrow z^{\prime}=\left(u^{\prime}, v^{\prime}\right), \quad \text { in } L_{\mathrm{loc}}^{q+1}\left(\mathbb{H}^{N}\right) \times L_{\mathrm{loc}}^{p+1}\left(\mathbb{H}^{N}\right), 2 \leq p, q<\frac{N+2}{N-2} .
\end{gathered}
$$

Moreover, $z^{\prime}=\left(u^{\prime}, v^{\prime}\right)$ solves equation (1.1).

Now we want to prove that $z^{\prime} \neq 0$. If it is not true, then we have $z^{\prime}=\left(u^{\prime}, v^{\prime}\right)=(0,0)$. We claim that: For any $1>r>2-\sqrt{3}$,

$$
\int_{\mathbb{\mathbb { N } ^ { N }} \cap\{|x| \geq r\}}\left[\left(u_{n}^{\prime}\right)_{+}\right]^{q+1} d V_{\mathbb{M}^{N}}=o(1), \quad \int_{\mathbb{W}^{N} \cap\{|x| \geq r\}}\left[\left(v_{n}^{\prime}\right)_{+}\right]^{p+1} d V_{\mathbb{\mathbb { M } ^ { N }}}=o(1) .
$$

To do this, let us fix a point $a \in S_{\sqrt{3}}$. Let $\Phi \in C_{0}^{\infty}(A(a, \sqrt{3}))$ such that $0 \leq \Phi \leq 1$, where $A(a, \sqrt{3})=B(a, \sqrt{3}) \cap \mathbb{W}^{N}$, where $B(a, \sqrt{3})$ is the Euclidean ball with centre $a$ and radius $\sqrt{3}$ and

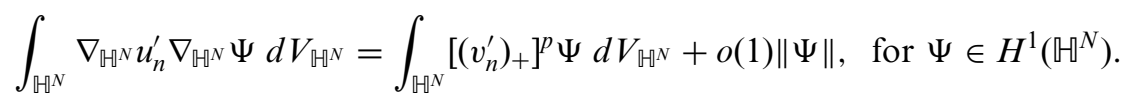

Now putting $\Psi=\Phi^{2}\left(u_{n}^{\prime}\right)_{+}$in the above identity, we get

$$
\int_{\mathbb{\mathbb { H } ^ { N }}} \nabla_{\mathbb{\mathbb { N } ^ { N }}} u_{n}^{\prime} \nabla_{\mathbb{\boxplus}^{N}}\left[\Phi^{2}\left(u_{n}^{\prime}\right)_{+}\right] d V_{\mathbb{U}^{N}}=\int_{\mathbb{\boxplus}^{N}}\left[\left(v_{n}^{\prime}\right)_{+}\right]^{p} \Phi^{2}\left(u_{n}^{\prime}\right)_{+} d V_{\mathbb{\boxplus}^{N}}+o(1) .
$$

A simple computation gives

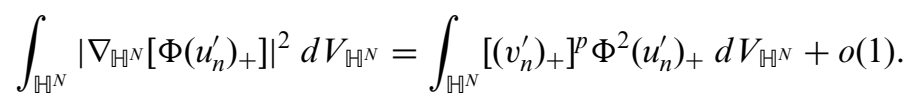

Now using (5.2), the Hölder inequality and the Poincarè-Sobolev inequality, we get

$$
\begin{aligned}
& \left\|\Phi\left(u_{n}^{\prime}\right)_{+}\right\|_{H^{1}\left(\mathbb{H}^{N}\right)}^{2} \\
& \quad \leq\left(\int_{\mathbb{H}^{N}}\left[\Phi\left(v_{n}^{\prime}\right)_{+}\right]^{p+1} d V_{\mathbb{H}^{N}}\right)^{\frac{1}{p+1}}\left(\int_{\mathbb{H}^{N}}\left[\Phi\left(u_{n}^{\prime}\right)_{+}\right]^{p+1} d V_{\mathbb{H}^{N}}\right)^{\frac{1}{p+1}}\left(\int_{A(a, \sqrt{3})}\left[\left(v_{n}^{\prime}\right)_{+}\right]^{p+1} d V_{\mathbb{H}^{N}}\right)^{\frac{p-1}{p+1}} \\
& \leq S_{N, p}^{-2}\left\|\Phi\left(v_{n}^{\prime}\right)_{+}\right\|_{H^{1}\left(\mathbb{H}^{N}\right)}\left\|\Phi\left(u_{n}^{\prime}\right)_{+}\right\|_{H^{1}\left(\mathbb{H}^{N}\right)}\left(\int_{A(a, \sqrt{3})}\left[\left(v_{n}^{\prime}\right)_{+}\right]^{p+1} d V_{\mathbb{H}^{N}}\right)^{\frac{p-1}{p+1}} .
\end{aligned}
$$

Similarly,

$$
\begin{aligned}
& \left.\left\|\Phi\left(v_{n}^{\prime}\right)_{+}\right\|_{H^{1}(\mathbb{M} N}^{2}\right)
\end{aligned}
$$

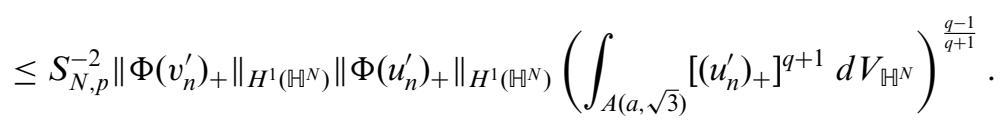


Now, if $\left\|\Phi\left(v_{n}^{\prime}\right)_{+}\right\|_{H^{1}\left(\mathbb{H}^{N}\right)} \not \rightarrow 0$ and $\left\|\Phi\left(u_{n}^{\prime}\right)_{+}\right\|_{H^{1}\left(\mathbb{H}^{N}\right)} \not \rightarrow 0$ as $n \longrightarrow \infty$, we get

$$
\begin{aligned}
S_{N, p}^{4} & \leq\left(\int_{A(a, \sqrt{3})}\left[\left(v_{n}^{\prime}\right)_{+}\right]^{p+1} d V_{\mathbb{\llbracket} \mathbb{N}^{N}}\right)^{\frac{p-1}{p+1}}\left(\int_{A(a, \sqrt{3})}\left[\left(u_{n}^{\prime}\right)_{+}\right]^{q+1} d V_{\mathbb{\boxplus}^{N}}\right)^{\frac{q-1}{q+1}} \\
& <\delta_{1}^{\frac{p-1}{p+1}} \delta_{2}^{q-1} \\
& <S_{N, p}^{4},
\end{aligned}
$$

which is a contradiction. This implies that $\int_{\mathbb{H}^{N}}\left[\Phi\left(v_{n}^{\prime}\right)_{+}\right]^{p+1} d V_{\mathbb{G}^{N}} \rightarrow 0$ and $\int_{\mathbb{G}^{N}}\left[\Phi\left(u_{n}^{\prime}\right)_{+}\right]^{q+1} d V_{\mathbb{N}^{N}} \rightarrow 0$. Since $a \in S_{\sqrt{3}}$ is arbitrary, the claim follows.

If $1<p, q<\frac{N+2}{N-2}$, this together with the fact that

$$
z_{n}^{\prime}=\left(u_{n}^{\prime}, v_{n}^{\prime}\right) \longrightarrow(0,0), \quad \text { in } L_{\mathrm{loc}}^{q+1}\left(\mathbb{H}^{N}\right) \times L_{\mathrm{loc}}^{p+1}\left(\mathbb{H}^{N}\right), \quad 2 \leq p, q<\frac{N+2}{N-2},
$$

immediately gives a contradiction to (5.1). It implies that $z^{\prime}=\left(u^{\prime}, v^{\prime}\right) \neq(0,0)$. It is easy to see that $I^{\prime}\left(z^{\prime}\right)=0$ by the fact that $\left\{z_{n}^{\prime}\right\}$ is a $(P S)_{c}$-sequence and $I^{\prime}\left(z_{n}^{\prime}\right) \rightarrow 0$ in $E^{*}$ as $n \rightarrow \infty$. Thus, $z^{\prime}$ is a positive solution of (1.1).

By Lemma 5.4, we know that the set

$$
\Lambda:=\left\{(u, v) \in E=H^{1}\left(\mathbb{H}^{N}\right) \times H^{1}\left(\mathbb{H}^{N}\right): I^{\prime}(u, v)=0,(u, v) \not \equiv(0,0)\right\}
$$

is a non-empty set. We also defined that a ground state is a minimizer of the variational problem

$$
I^{\infty}=\inf \{I(u, v) \mid(u, v) \in \Lambda\}
$$

Proof of Theorem 1.5. Proving as in Proposition 4.1, and taking $(u, v) \in H^{1}\left(\mathbb{H}^{N}\right) \times$ $H^{1}\left(\mathbb{M}^{N}\right)$ and $I^{\prime}(u, v)=0$, we also have $\|u\|_{L^{q+1}\left(\mathbb{M}^{N}\right)} \geq C>0$ and $\|v\|_{L^{p+1}\left(\mathbb{M}^{N}\right)} \geq C>0$.

Suppose now that $z_{n}=\left(u_{n}, v_{n}\right) \in H^{1}\left(\mathbb{M}^{N}\right) \times H^{1}\left(\mathbb{M}^{N}\right)$ is a minimizing sequence of $I^{\infty}$, that is

$$
I\left(z_{n}\right) \longrightarrow I^{\infty}, I^{\prime}\left(z_{n}\right)=0, z_{n} \neq 0 .
$$

Clearly, $\left\{z_{n}\right\}$ is a $(P S)_{I^{\infty}}$ sequence for $I$, and by Lemma 5.4, we see that $\left\{z_{n}\right\}$ is uniformly bounded in $H^{1}\left(\mathbb{H}^{N}\right) \times H^{1}\left(\mathbb{H}^{N}\right)$ and there is a critical point $\left(u_{1}, v_{1}\right)$ of $I$ with least critical value, which is a ground state solution of problem (1.1). Moreover, we have $I\left(u_{1}, v_{1}\right)>0$ and $\left(u_{1}, v_{1}\right)>(0,0)$.

ACKNOWLEDGEMENTS. This work was supported by the National Natural Sciences Foundations of China(Nos. 11201140 and 11271120), Hunan Provincial Natural Science Foundation of China (No. 14JJ6005) and Program for excellent talents in Hunan Normal University (No. ET12101). The author would like to thank the referees for their valuable comments and suggestions.

\section{REFERENCES}

1. A. D. Alexandrov, Uniqueness theorems for surfaces in the large V, Amer. Math. Soc. Trans. 21 (1962), 412-416. 
2. L. Almeida, L. Damascelli and Y. Ge, A few symmetry results for nonlinear elliptic PDE on non-compact manifolds, Ann. Inst. Henri Poincaré Anal. Non-Linîaire 19 (2002), 313-342.

3. L. Almeida and Y. Ge, Symmetry result for positive solutions of some elliptic equations on manifolds, Ann. Global Anal. Geom. 18 (2000), 153-170.

4. H. Berestycki and P. L. Lions, Nonlinear scalar field equations (I), Arch. Ration. Mech. Anal. 82 (1983), 313-376.

5. M. Bhakta and K. Sandeep, Poincarè Sobolev equations in the hyperbolic space, Calc. Var. Partial Differ. Equ. 44 (2012), 247-269.

6. M. Bonforte, F. Gazzola, G. Grillo and J. L. Vàzquez, Classification of radial solutions to the Emden-Fowler equation on the Hyperbolic space, Calc. Var. PDE, 46(1-2) (2013), 375401. arXiv:1104.3666v2.

7. J. Busca and R. Manasevich, A Liouville-type theorem for Lane-Emden systems, Indiana Univ. Math. J. 51 (2002), 37-51.

8. J. Busca and B. Sirakov, Symmetry results for semi-linear elliptic systems in the whole space, J. Differ. Equ. 163 (2000), 41-56.

9. L. Caffarelli, B. Gidas and J. Spruck, Asymptotic symmetry and local behavior of semilinear elliptic equations with critical Sobolev growth, Commun. Pure Appl. Math. XLII (1989), 271-297.

10. W. Chen and C. Li, Classification of solutions of some nonlinear elliptic equations, Duke Math. J. 63 (1991), 615-622.

11. D. G. De Figueiredo and P. Felmer, A Liouville-type theorem for elliptic systems, Ann. Sci. Norm. Sup. Pisa 21 (1994), 387-397.

12. D. G. De Figueiredo and J. Yang, Decay, symmetry and existence of solutions of semilinear elliptic systems, Nonlinear Anal. 33 (1998), 211-234.

13. B. Gidas, W. M. Ni and L. Nirenberg, Symmetry and related properties via the maximum principle, Commun. Math. Phys. 68 (1979), 209-243.

14. B. Gidas and J. Spruck, A priori bounds for positive solutions of nonlinear elliptic equations, Commun. Partial Differ. Equ. 6 (1981), 883-901.

15. B. Gidas and J. Spruck, Global and local behavior of positive solutions of nonlinear elliptic equations, Commun. Partial Differ. Equ. 17 (1992), 923-940.

16. E. Hopf, Lectures on differential geometry in the large, Seminar lectures New York University and Stanford University (Springer, New York, NY, 1956).

17. Q. Jie, A priori estimates for positive solutions of semi-linear elliptic systems, J. Partial Differ. Equ. 1 (1988), 525-598.

18. H. E. Lions and E. Magen, Nonhomogeneous boundary value problems and applications, vol I. ( Springer-Verlag, Berlin, Germany, 1972).

19. P. L. Lions, The concentration-compactness principle in the calculus of variations. The locally compact case, part 2, Annales de l'institut Henri Poincaré Analyse non linéaire 1 (1984), 223-283.

20. S. Li and M. Willem, Applications of local linking to critical point theory, J. Math. Anal. Appl. 189 (1995), 6-32.

21. G. Li and J. Yang, Asymptotically linear elliptic systems, Commun. Partial Differ. Equ. 29 (2004), 925-954.

22. G. Mancini and K. Sandeep, On a semi-linear elliptic equaition in $\mathbb{\boxplus}^{N}$, Ann. Sci. Norm. Super. Pisa Cl. Sci 7 (2008), 635-671.

23. E. Mitidieri, Non-existence of positive solutions of semi-linear elliptic systems in $R^{N}$, Differ. Int. Equ. 9 (1996), 465-479.

24. J. Serrin and H. Zou, Non-existence of positive solutions of Lane-Emden systems, Differ. Int. Equ. 9 (1996), 635-653.

25. J. Serrin, A symmetry problem in potential theory, Arch. Ration. Mech. Anal. 43 (1971), 304-318.

26. M. A. Souto, Sobre a existencia de solucoes positivas para sistemas cooperativos nao lineares, $\mathrm{PhD}$ Thesis (Unicamp, Campinas - SP, Brazil, 1992).

27. H. Triebel, Theory of function spaces II, Springer Monographs in Mathematics, vol. 84 (Birkhäuser, Heidelberg, Germany, 1992).

28. J. Wolf, Spaces of constant curvature (McGraw-Hill, New York, NY, 1967). 